\title{
Venus flytrap carnivorous lifestyle builds on herbivore defense strategies
}

\author{
Felix Bemm, ${ }^{1,8}$ Dirk Becker, ${ }^{2}$ Christina Larisch, ${ }^{2}$ Ines Kreuzer, ${ }^{2}$ Maria Escalante-Perez, ${ }^{2}$ \\ Waltraud X. Schulze, ${ }^{3}$ Markus Ankenbrand, ${ }^{1,4}$ Anna-Lena Van de Weyer, ${ }^{1,8}$ \\ Elzbieta Krol, ${ }^{2}$ Khaled A. Al-Rasheid, ${ }^{2,5}$ Axel Mithöfer, ${ }^{6}$ Andreas P. Weber, ${ }^{7}$ \\ Jörg Schultz, ${ }^{1}$ and Rainer Hedrich ${ }^{2}$
}

${ }^{1}$ Center for Computational and Theoretical Biology, Campus Hubland Nord; Department of Bioinformatics, Biocenter, Am Hubland, University of Würzburg, D-97218 Würzburg, Germany; ${ }^{2}$ Institute for Molecular Plant Physiology and Biophysics, Biocenter, University of Würzburg, 97082 Würzburg, Germany; ${ }^{3}$ Department of Plant Systems Biology, University of Hohenheim, 70593 Stuttgart, Germany; ${ }^{4}$ Department of Animal Ecology and Tropical Biology, Biocenter, Am Hubland, 97074 Würzburg, Germany; ${ }^{5}$ Zoology Department, College of Science, King Saud University, Riyadh 11451, Saudi Arabia; ${ }^{6}$ Bioorganic Chemistry Department, Max-Planck-Institute for Chemical Ecology, 07745 Jena, Germany; ${ }^{7}$ Institute of Plant Biochemistry, Cluster of Excellence on Plant Sciences (CEPLAS), Heinrich-Heine-University, 40225 Düsseldorf, Germany

\begin{abstract}
Although the concept of botanical carnivory has been known since Darwin's time, the molecular mechanisms that allow animal feeding remain unknown, primarily due to a complete lack of genomic information. Here, we show that the transcriptomic landscape of the Dionaea trap is dramatically shifted toward signal transduction and nutrient transport upon insect feeding, with touch hormone signaling and protein secretion prevailing. At the same time, a massive induction of general defense responses is accompanied by the repression of cell death-related genes/processes. We hypothesize that the carnivory syndrome of Dionaea evolved by exaptation of ancient defense pathways, replacing cell death with nutrient acquisition.
\end{abstract}

[Supplemental material is available for this article.]

Carnivory is not restricted to the realm of animal life; the plant kingdom also has flesh eaters (Darwin 1875; Adamec 1997; Krol et al. 2012). This remarkable trait has evolved independently at least six times in plants (Albert et al. 1992; Ellison and Gotelli 2009). Techniques to catch prey are as diverse as pitcher, sticky, and snap traps. Remarkably, some of these have evolved convergently in different clades. However, the carnivory lifestyle appears costly for the plant (Givnish et al. 1984), implying that the metabolic price deters a more frequent use of plant carnivory than its invention. Assuming that plant carnivory is based on readily accessible traits, Charles Darwin suggested routes as to how plant carnivory was gradually acquired (Darwin 1875). Nonetheless, despite increasing insights into the phylogeny of the carnivorous plants, current models mainly focus on the morphological and anatomical traits of the typical organs. Knowledge about the molecular and physiological roots of carnivory is essentially lacking, as are unbiased, data-driven approaches aimed at unraveling the molecular mechanisms involved in plant carnivory.

Here, we focus on one of the most prominent carnivorous plants, Dionaea muscipula, which belongs to the Caryophyllales order. At least four of the carnivorous families-Nepenthaceae,

\footnotetext{
${ }^{8}$ Present address: Max-Planck-Institute for Developmental Biology, Department of Molecular Biology, 72076 Tübingen, Germany Corresponding author: hedrich@botanik.uni-wuerzburg.de Article published online before print. Article, supplemental material, and publication date are at http://www.genome.org/cgi/doi/10.1101/gr.202200.115. Freely available online through the Genome Research Open Access option.
}

Drosophyllaceae, Dioncophyllaceae, and Droseraceaea-can be unequivocally placed within the Caryophyllales (Heubl et al. 2006). Their presence within a single clade indicates a common ancestor and argues that sticky traps are the most ancient trap form within this clade; the snap traps found in Dionaea and Aldrovanda are derived from a sticky trap. Current phylogenetic reconstructions indicate that this type of trap evolved only once within the common ancestor of these two species (Cameron et al. 2002). Today, the Venus flytrap, D. muscipula, is only found natively in the Green Swamp of North and South Carolina. The leaf at the end of a photosynthetically active petiole of Dionaea develops into a green bilobed snap trap, with the inner trap surface equipped with peculiar mechanosensitive hairs. These hairs allow Dionaea to recognize prey by transducing a mechanical stimulation into an electrical signal known as action potential (AP). The first mechano-electric stimulation of a trigger hair sets the trap to an "attention mode." In other words, a one touch-induced AP is memorized but does not close the trap. With a second AP, the Dionaea trap closes within a fraction of a second (Forterre et al. 2005; Escalante-Perez et al. 2014), locking the prey between the two trap lobes. Prey, when trying to escape, repeatedly touch the mechano-sensors, thereby eliciting repetitive firing of APs. In a very recent study, Böhm et al. (2016b) showed that the Venus flytrap can count the number of APs generated, thus "memorizing" how often an insect has touched it and preventing false alarms.

(c) 2016 Bemm et al. This article, published in Genome Research, is available under a Creative Commons License (Attribution-NonCommercial 4.0 International), as described at http://creativecommons.org/licenses/by-nc/4.0/. 
While two APs trigger fast trap closure, more than five APs result in the capture organ becoming hermetically sealed. Numerous glands that cover the inner surface of the stomach start expressing genes that encode enzymes involved in decomposing the prey into its nutrient building blocks (Schulze et al. 2012). Interestingly, mechano-electric stimulation can be substituted by direct administration of the touch hormone jasmonic acid (JA), suggesting that the number of APs translates into a chemical signal that roughly informs the plant about the size and nutrient content of a struggling prey.

Although Darwin recognized early on that Dionaea's animal prey consumption is based on electrical excitability (BurdonSanderson 1872) and fast flytrap biomechanics, the molecular mechanism of these animal-like features still remains poorly understood. Despite recent efforts in the molecular analysis of various carnivorous plant species, no lifestyle-specific genes have as yet been identified (Ibarra-Laclette et al. 2011, 2013; Leushkin et al. 2013; Fleischmann et al. 2014; Barta et al. 2015; Cao et al. 2015; Carretero-Paulet et al. 2015a,b; Stephens et al. 2015; Tran et al. 2015). To gain mechanistic insights into the molecular processes underlying the carnivory syndrome, we combined ultrastructural, physiological, and proteomic analyses with thorough transcriptome sequencing to analyze the prey-dependent changes in gross gene expression patterns and nutrient transport in relation to the endocrine biology of the glands.

\section{Results}

\section{The molecular composition of the trap}

Although the Venus flytrap is uniquely adapted to circumvent malnutrition on poor soils, it still shows the typical morphological and gross physiological properties of a green plant. Nonetheless, Dionaea has evolved a highly specialized trap for hunting animals (Volkov et al. 2013), and profound morphological features distinguish the trap-forming leaf tip from the leaf base (petiole). These features can be attributed to trap-specific functions, namely, the need to catch and digest prey and, subsequently, the absorption of released nutrients. We hypothesized that these functional traits should relate to the nature and the abundance of transcripts specific to traps. Consequently, we compared the transcriptome of the trap with the overall transcriptomic landscape of D. muscipula, covering root, flower, and petiole tissue. Furthermore, we analyzed the transcriptomes of nonstimulated traps and glands versus those of traps and glands processing insects or stimulated with coronatine (COR), a molecular mimic of the phytohormone JA. In total, 6.5 billion reads (Supplemental Table S1) were produced using high-throughput RNA sequencing on the Illumina HiSeq platform. Following assembly, correction, and filtering, we found that the final transcriptome of the Venus flytrap comprised 51,196 potential genes. Assessment of the completeness of the reference transcriptome with Benchmarking Universal Single-Copy Orthologs (BUSCO) (Simao et al. 2015) revealed that 91\% of all universal single-copy orthologs in the BUSCO's plantae benchmark set were present and complete. A conservative high-confidence ortholog assignment with CRB-BLAST (Aubry et al. 2014) resulted in 19,293 genes with a nonambiguous ortholog in the Arabidopsis thaliana reference genome.

By using this data set as a reference transcriptome, we aimed to unravel the molecular adaptations associated with prey capture and processing in the Dionaea leaf. Integrating data from flowers, roots, and petioles into this analysis, we identified general trends that characterize these organs using a principal component analysis (PCA) on variance-stabilized expression data for all 51,196 genes (Fig. 1A; Supplemental Table S2). The component with the largest spread (34.87\%) separated the photosynthetically active petiole and trap from the root and the flower. The second largest spread component $(22.08 \%)$ divided organs into reproductive (flower) and nonreproductive (trap, petiole, and root) organs. The third strongest component (15.50\%) distinguished the trap from all the other tissues (Supplemental Fig. S1). Out of 2139 genes significantly contributing to the third component, only 540 $(25,24 \%)$ were annotated with a gene ontology (GO) term. These genes were associated with responses to external stimuli and the generation of precursor metabolites and energy (Supplemental Table S2). Due to the pronounced ratio of nonannotated genes $(74,76 \%)$, we could not further link the third component to other particular features at this point. To analyze the transcriptomic commonalities underlying the observed separations, respectively groupings, of the first three components more precisely, we identified differentially expressed genes (DEGs) in the root, trap, flower, and petiole using an all-versus-all pairwise comparison. From this analysis, we identified 17,715 out of the 51,196 genes as differentially expressed in at least one pairwise comparison, while 14,089 DEGs were detected in multiple different comparisons (Fig. 1B). A Venn analysis revealed 3626 organ-specific DEGs, with the highest number for the root (1470) followed by the flower (1113), petiole (655), and trap (388). However, flowers only exhibited organ-specific DEGs enriched for biological functions related to flower physiology, namely, anatomical structure morphogenesis, cell growth, and the cell cycle (Supplemental Table S3). Petioles, roots, and traps showed no obvious organ-specific signatures. The results of the PCA and the Venn analysis allowed us to speculate that the transcriptomic landscape of a nonstimulated trap is represented by a patchwork of genes that are also expressed in other organs. A global correlation measurement based on normalized expression counts demonstrated that the expression profile of nonstimulated traps strongly correlates with that of the petioles (Fig. 1C). Thus, our results provide the first transcriptome-wide molecular support for the common assumption that the trap is indeed a modified leaf.

Since the overall trap transcriptome integrates the expression profile of various typical leaf cell types, we could not exclude the existence of carnivorous signatures in certain trap-specific cell types such as glands. Considering that prey digestion and nutrient uptake are directly linked to the activity of about 37,000 glands on the inner surface of the trap (Juniper et al. 1989; Escalante-Perez et al. 2011), we generated a transcriptomic profile of isolated nonstimulated glands, as well as the trap rim, which is free of digesting glands, to clarify this issue. Correlating the resulting expression profiles of the gland, rim, root, and petiole revealed that the rim is most akin to the petiole, and glands showing highest correlation with the roots (Fig. 1D). Testing for differential expression in glands and roots compared with petioles revealed 7427 DEGs in glands and 6897 in roots. Both tissues shared 5013 DEGs exhibiting strong signals of transport, stress response, and protein metabolic processes, according to the GO. A detailed study of the underlying molecular functions using lower level MapMan bins (level 3) (Thimm et al. 2004), coupled with a generally applicable gene-set enrichment (GAGE) (Luo et al. 2009), revealed that DEGs from both tissues were enriched for gene sets involved in transcriptional regulation via AP2, C2H2, WRKY, and bHLH-like transcription factors, protein synthesis (ribosome biogenesis), protein modification, and degradation, as well as protein targeting 
A

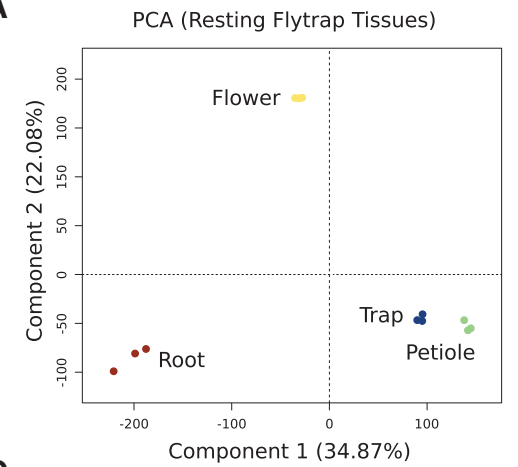

C

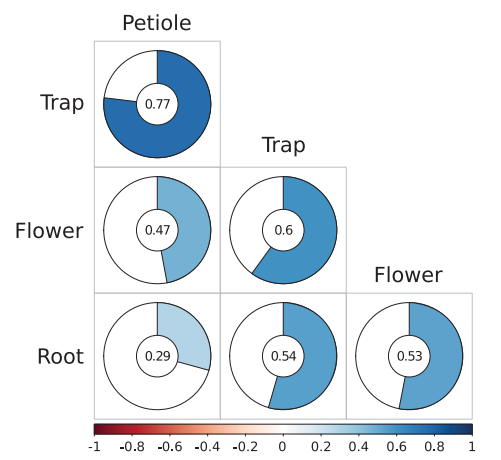

B

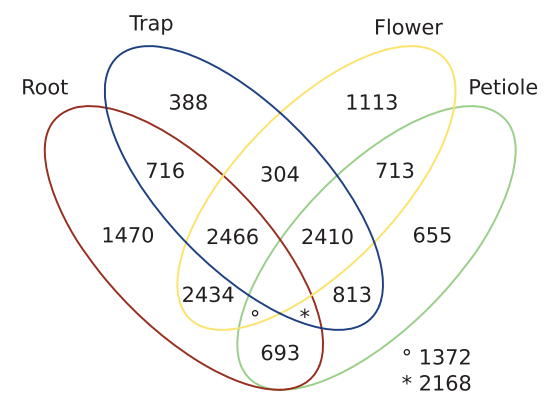

D

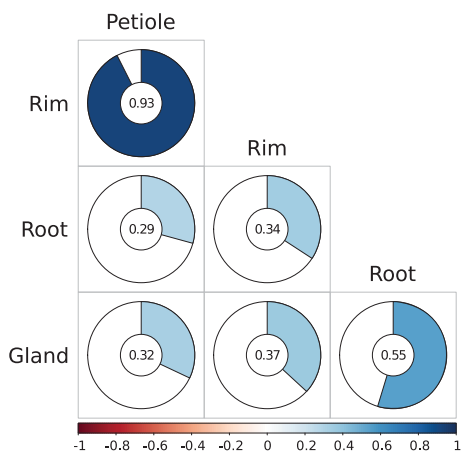

Figure 1. The transcriptomic landscape of the nonstimulated Venus flytrap (Dionaea muscipula). $(A$ ) Principal component analysis of all biological replicates $(n=3)$ from petiole, trap, root, and flower. The first two dimensions account for $57 \%$ of all the variance in the nonstimulated Venus flytrap data (for additional dimensions, see Supplemental Fig. S1). (B) Venn diagram from an all-versus-all differential expression analysis. Overall, 14,744 DEGs were shared by at least two tissues, while 3626 DEGs are most likely expressed in a tissue-specific manner. (C) Hierarchically clustered visualization of the global Pearson correlation between all major organs. All individual pairwise correlations are significant according to multiple testing adjusted probabilities $(P \leq 0.01)$. (D) Visualization of the global Pearson correlation between all major organs with traps being represented by rim and gland. Again all correlations tested are significant $(P \leq 0.01)$.

along the secretory pathway (Supplemental Table S4). The observed similarity of glands and roots is therefore based on gene expression profiles that reflect the heterotrophic physiology and secretory capability of both tissues.

\section{Gland functional morphology}

Our finding that glands show a gene expression profile reminiscent of heterotrophic secreting tissues prompted us to analyze the gland structure in more detail (Fig. 2). Early morphological studies show that each gland is composed of 46 cells (Juniper et al. 1989). Transmission electron microscopy (TEM) shows the typical three-layer arrangement of a gland (Fig. 2C). Two basal cells are surmounted by four endodermoid (stalk) cells, comprising layer 3 (L3, blue). Overlying this is an inner layer (L2, brown) formed by eight cells, followed by an outer layer (L1, green) of 32 secretory cells that form the head of a mature gland (Lloyd 1942; Rea et al. 1983). All gland cells are connected by plasmodesmata, thus creating a metabolic and ionic cytoplasmic continuum. Both the cell wall and the cuticle of the glands are less developed and more permeable to solutes than are the common epidermal cells (Joel et al. 1983). In nonstimulated glands, the innermost stalk cells (L3) are tightly packed with oleosomes (Fig. 2F), implying that a triacylglycerol (TAG) reservoir represents the primary currency for energy consumptive processes. Supporting this observation is our finding that transcripts involved in TAG breakdown, includ-

ing peroxisomal beta-oxidation, are highly expressed in glands (Supplemental Table S5). Highly abundant mitochondria in L2 cells (Fig. 2E) suggest that activated fatty acids provide the dominant resource for ATP generation at this site. The pronounced appearance of plasma membrane invaginations at the basal ends of L1 and in L2 cells (Fig. $2 \mathrm{D}, \mathrm{E})$ is indicative of an increased interface capacity for nutrient recognition and transport (see below). Finally, the existence of an expanded rough endoplasmic reticulum in L1 together with pronounced transcriptional activity in glands indicates that a highly dynamic protein biosynthesis and translocation machinery is present in the outer cell layer even when in the nonstimulated state (see Fig. 2D and below).

In nature, gland secretion is stimulated upon insect capture and, in the laboratory, by the application of JA or its molecular mimic COR (Radhika et al. 2010). Both stimuli result in gross ultrastructural changes in gland cells. In L1 cells, gland stimulation results in the dispersal of large apical vacuoles (Supplemental Fig. S2). Structural changes in head cells of the L2 layer are even more dramatic. Glands of insect digesting traps exhibit L2 cells containing large vacuoles (probably generated through vesicle fusions) and a heavily invaginated plasma membrane that completely covers the stimulated cells and is indicative of active secretion processes. In line with this observation, we have previously shown that upon stimulation with COR, glands change their surface-to-volume ratio by increasing their mean surface area by $>30 \%$ (Escalante-Perez et al. 2011).

\section{Active glands in secretion mode}

To associate the ultrastructural changes in glands with cellular activities, we performed a comparative transcriptomic analysis of nonstimulated and active glands. Glands were stimulated with insects so as to activate secretion, and stimulated glands were mechanically separated from the inner trap surface $24 \mathrm{~h}$ after stimulus onset and subjected to transcriptome profiling. Stimulated glands show 3447 genes up-regulated and 2826 genes down-regulated. The majority of the up-regulated genes are indicative of highly active transport, signal transduction, and stress responses (Supplemental Table S6). In detail, transcriptional regulators for stress responses together with components of the secretory pathway, including pathogen-related proteins, cysteine, and serine proteases contributed to the strong stress signal.

The combination of profile-based signal peptide annotations with high-throughput screening (HTS) proteomics data from the digestive fluid of the "green stomach" allowed the Venus flytrap secretome to be characterized in more detail. Proteomics data were generated for traps stimulated with either insect, COR, or trigger hair bending (mechanical stimulation). Within the group of

\section{Genome Research}

www.genome.org 


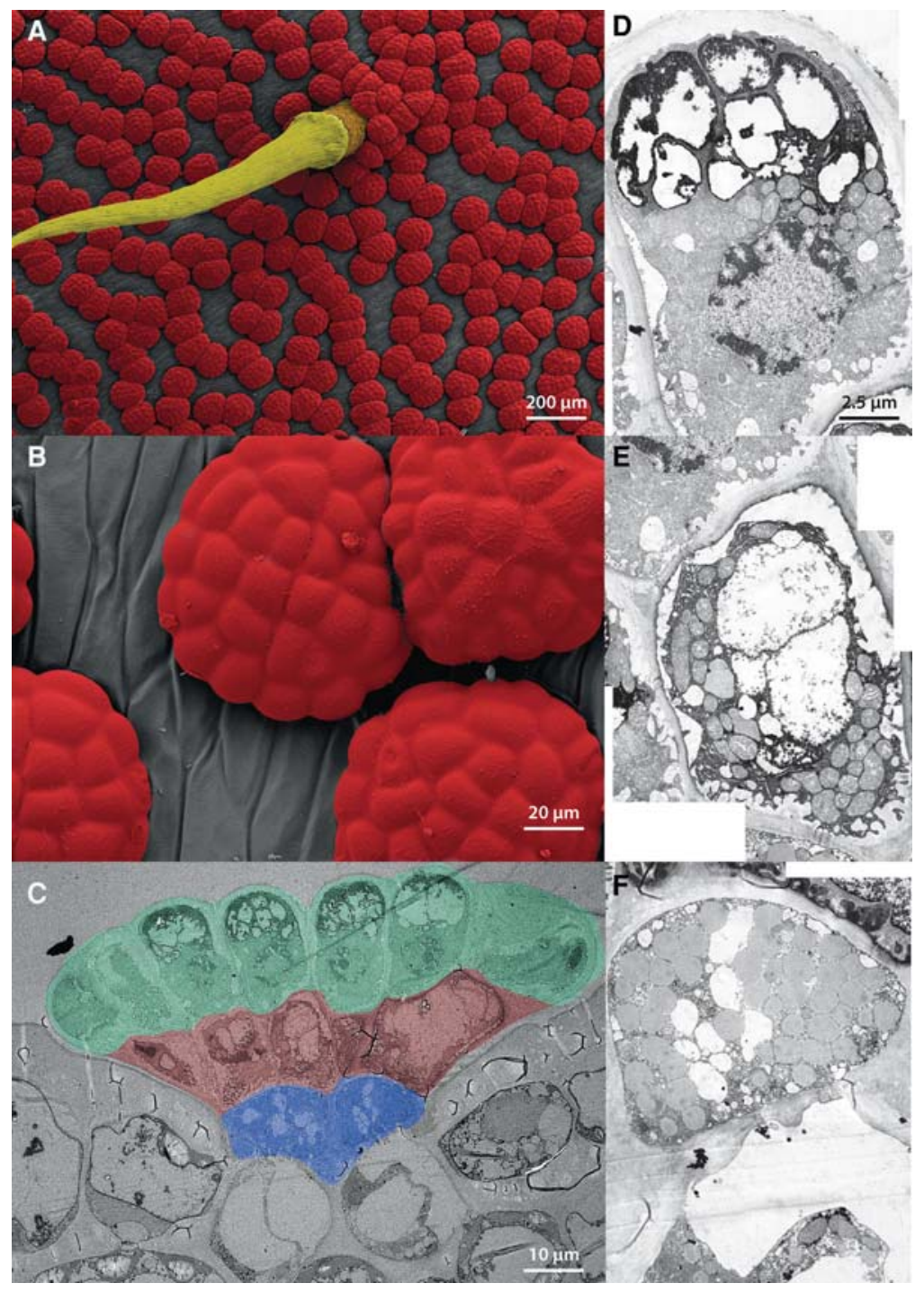

Figure 2. Gland functional morphology. ( $A$ ) REM false-colored micrograph showing a trigger hair (yellow) surrounded by multiple glands (red) on the inner surface of a Dionaea trap. (B) Close-up of glands (from $A)$. ( $(C-F)$ TEM micrographs of gland sections. Structural organization of a gland $(C)$ consisting of three functional layers (L1-L3): outer layer L1 (green), inner layer L2 (brown), and endodermoid layer L3 (blue). Secretory cell of the $\mathrm{L} 1$ outer layer $(D)$ characterized by large vacuoles and the presence of rough endoplasmic reticulum. Cell of the inner L2 layer $(E)$ exhibiting numerous plasma membrane invaginations. Beta-oxidation probably occurs in L2 cells, which contain big central vacuoles, numerous peroxisomes, and a remarkable number of mitochondria (see also Supplemental Fig. S2). This implies the existence of energy demanding, metabolically active, processes in L2 cells. Endodermoid (stalk) cell $(F)$ comprising layer L3 harboring plenty of oleosomes.

up-regulated, signal sequence containing transcripts, we identified 42 highly abundant DEGs. By using the HTS proteomic data set of the secretion fluid, we independently confirmed that these 42 candidate genes are indeed actively secreted (Fig. 3A, left; Supplemental Table S7). Among them, 15 proteins were secreted irrespective of the nature of the stimulus (COR, trigger hair stimulation, or insect). They encoded secretome-related proteins with hydrolase activity such as proteases, phosphatases, and chitinases, as well as defensin-like (DEFL) cysteine-rich proteins (Fig. 3A, right; Supplemental Table S7). In contrast, only 13 down-regulated DEGs had proteomic evidence together with an annotated signal peptide (Fig. 3A, denominators; Supplemental Table S7). Among these, we found transcripts encoding the Nepenthesin-like aspartic protease
(Buch et al. 2015) and a Lipid Transfer Protein (LTP). Both these genes were already highly expressed in nonstimulated glands, but the majority of hydrolase genes showed a massive induction ranging from fivefold to 900 -fold following stimulation (Fig. 3A, right).

To gain insights into the kinetics of the secretion process, we followed the time course of selected marker hydrolases in traps, namely, SAG12 and SCPL49 proteases using qPCR. COR-stimulated traps showed rapid transcriptional activation of hydrolases 1-2 $\mathrm{h}$ after stimulus onset. As Paszota et al. (2014) have already shown for VF CHITINASE I, SAG12 and SCPL49 hydrolase expression reached peak levels at $24-48 \mathrm{~h}$ after stimulation, irrespective of the nature of the stimulus (insect, trigger hair bending/mechanics, or COR) (Fig. 3B). To further investigate the functional interaction between mechano- and chemo-sensing during prey capture, we performed a kinetic analysis of hydrolase activation in response to two consecutive stimuli (Fig. 3C). Traps were first stimulated mechanically. This was followed by a second stimulus $8 \mathrm{~h}$ later, applied either as a second trigger hair stimulation or chemically by means of chitin application (a component of the insect coat). The first mechanical stimulation of the trap's trigger hairs resulted in a strong increase in VF CHITINASE I expression after $8 \mathrm{~h}$ (Escalante-Perez et al. 2011; Schulze et al. 2012; Scherzer et al. 2013). VF CHITINASE I expression remained elevated until $24 \mathrm{~h}$ but returned to its resting level after $48 \mathrm{~h}$ if no further stimulus was applied. In contrast, a second mechanical stimulation at $8 \mathrm{~h}$ further increased the already high $V F$ CHITINASE I mRNA levels (413-fold) until $48 \mathrm{~h}$ after the initial stimulus. If the second stimulus was of a prey-associated chemical such as chitin, VF CHITINASE I expression after $48 \mathrm{~h}$ was boosted up to almost 2000-fold.

In noncarnivorous plants, chemical sensing often involves receptor-like kinases (RLKs), such as the chitin sensing LysM subfamily (Antolin-Llovera et al. 2012). By searching for members of the trap's sensory system, we identified 237 RLKs. Nonstimulated traps already differentially expressed 201 RLKs compared with petioles. Active traps and glands showed 63 and 134 RLKs differentially expressed, respectively, compared with their corresponding nonstimulated control (Supplemental Table S8). A Dionaea LysMtype chitin oligosaccharide-responsive CERK1-like kinase (Miya et al. 2007) was significantly up-regulated following gland insect stimulation, suggesting that glands might be able to assess the movement (mechanics), chemistry, and quality of the prey during progressive digestion and adjust the secretion process accordingly. 
A
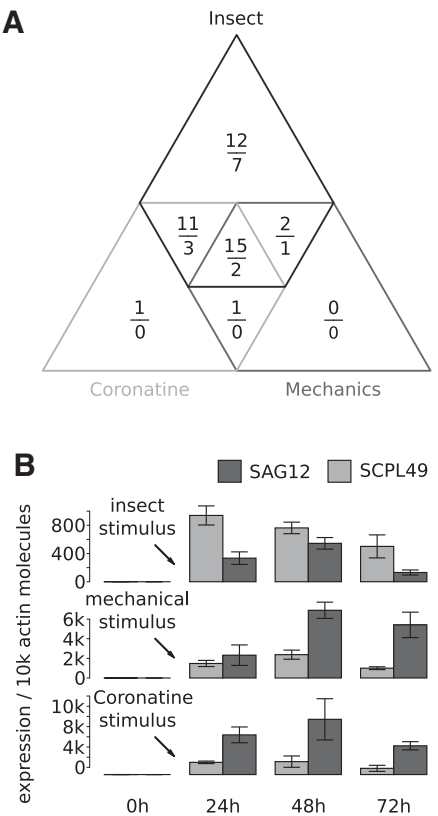

\begin{tabular}{l|l}
\hline Enzyme & FC \\
\hline Purple Acid Phosphatase 27 & 971 \\
S1/P1 Nuclease 1 & 802 \\
Cystein Peptidase C1A (SAG12) & 667 \\
Beta-Glucanase (BGL2) & 602 \\
Serine Carboxypeptidase 49 (SCPL49) & 496 \\
Ribonuclease T2 (RNS1) & 472 \\
Chitinase Class I (VF CHITINASE I) & 401 \\
Plant Peroxidase & 362 \\
S1/P1 Nuclease 2 & 340 \\
Plant Lipid Transfer Protein & 328 \\
Peptide-N4-Asparagine Amidase A & 26 \\
Pathogenesis-related Protein & 13 \\
LysM-containing Protein & 9 \\
Aspartic peptidase 1 & 7 \\
Aspartic peptidase 2 & 5 \\
\hline
\end{tabular}

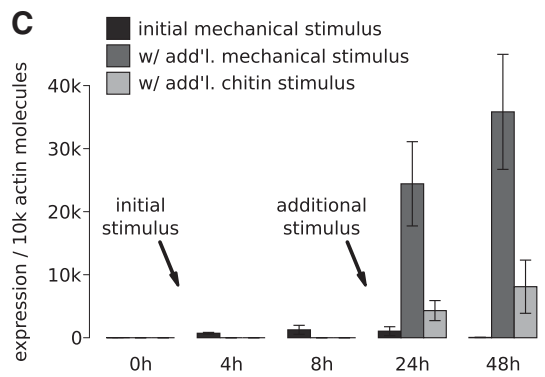

Figure 3. The Dionaea hydrolase cocktail is activated synergistically by touch and taste. ( $A$, left) Venn diagram of potential secretome members from an overlay of RNA-seq data (nonstimulated and insectstimulated glands) and HTS proteomic measurements of secreted fluid from chemically (COR), mechanically, and insect-stimulated traps. Numbers indicate up-regulated (top) and down-regulated (bottom) transcripts. Potential candidates are limited to transcripts that are differentially regulated, contain a proper signal peptide, and are detected at least once using HTS proteomics. $(A$, right) List of potential secretome members differentially up-regulated after insect stimulation and detected in all three HTS proteomic measurements. (B) qPCR time course of Dionaea marker hydrolases SCPL49 and SAG12 in response to insect, mechanical, and COR stimulation. Both enzymes are up-regulated several thousandfold after stimulation. Expression at time point $0 \mathrm{~h}$ is within statistical noise. (C) Synergistic stimulation of VFCHITINASE I expression in response to an initial mechanical stimulation followed by a chemical stimulus (chitin).

\section{Trap insect processing builds on JA-based touch/wound signaling}

Stimulus-induced hydrolase expression indicates that molecular pathways resembling defense and/or wounding responses accompany the carnivorous phenomenon. This hypothesis is corroborated by the strong stress-like response that prevails in stimulated glands (see above and Supplemental Table S6). To elucidate the system-wide response to insect feeding, we generated transcriptomic profiles of insect-activated traps, identifying 2137 genes as up-regulated, with 852 suppressed. Only a subset of 103 insect-stimulated DEGs were regulated in an insectspecific manner, and the majority of DEGs were differentially expressed in at least one other pairwise comparison of nonstimulated tissues.

Similar to the stimulated glands, insect-activated traps displayed a high transport activity together with a massive wounding response compared with nonstimulated tissues (Supplemental Table S9). Identified DEGs were mainly enriched for processes controlled or triggered by the stress hormones JA and abscisic acid, followed by ethylene, auxin, and salicylic acid. Corroborating previous findings that the wounding hormone JA plays an important role in carnivorous plants (Escalante-Perez et al. 2011; Nakamura et al. 2013; Libiakova et al. 2014), our analyses now demonstrate that not only JA biosynthesis but also its signaling components were differentially regulated. A detailed inspection showed that insect processing induces JA core components in traps
(Fig. 4A; Supplemental Table S10), and a highly similar transcriptional response was triggered when the secretion-inducing JA mimic COR was applied to glands and traps (Supplemental Tables S11, S12).

To obtain more detailed insight into JA signaling in active traps, we performed quantitative PCR (qPCR) to follow the kinetics of the JA receptor COI1 (CORONATINE INSENSITIVE 1) (Fig. 4B) and its coreceptor JAZ1 (JASMONIC ACID ZIM DOMAIN 1) (Fig. 4C; Chini et al. 2007; Wasternack and Hause 2013). COI1 is moderately expressed in nonstimulated traps, but within $4 \mathrm{~h}$ of either COR or insect stimulation, it is rapidly and sustainably repressed (Fig. 4B). In contrast to COI1, JAZ1 expression transiently increases in stimulated traps (12fold) (Fig. 4C), reaching peak expression after $4 \mathrm{~h}$ and remaining at sustained elevated levels for $24 \mathrm{~h}$. Both COR and insects seem to activate the oxylipin pathway that leads to the formation of 12-oxo-phytodienoic acid (OPDA). Insects, however, further promote biosynthesis of JA-Ile, the true COI1 ligand (see Fig. 4A; Sheard et al. 2010). These results indicate that insects mechanically induce JA-Ile signals, thereby shifting glands into the secretion mode. This finding is further substantiated by our observation that the JA antagonist coronatine-O-methyloxime (COR-MO), which prevents COI1-JAZ interaction leading to JAZ degradation, represses the mechanical induction of hydrolase expression (Fig. 4D).

Furthermore, the set of insect-stimulated DEGs in traps was comprised of genes related to the production of reactive oxygen species (ROS) (Supplemental Table S13) scavengers (Mittler et al. 2004) and components of the ER-quality-control machinery (ER-QC) (Supplemental Table S13; Liu and Howell 2010; Williams et al. 2014). Both processes are related to oxidative stress management and are essential for controlling protein translation and maturation (Howell 2013). These signals likely originate in active glands characterized by persisting translation, folding, and targeting of extracellular hydrolases (see above), as well as plasma membrane receptors and transporters (see below). Remarkably, we found 38 genes that are predicted to negatively regulate the programmed cell death (PCD) prevalent in active traps (Supplemental Table S14). Among these, we identified Dionaea HEXOKINASE 1 (HXK1), a negative regulator of PCD in higher plants (Kim et al. 2006; Bruggeman et al. 2015). Likewise, we detected a Dionaea homolog of NECROTIC SPOTTED LESIONS 1 (NSL1), a known suppressor of cell death programs and defense responses (Noutoshi et al. 2006). Furthermore, the cyclic nucleotide gated (CNG) type channel DEFENCE-NO-DEATH-1 (DND1), a key player in pathogen defense responses and PCD in Arabidopsis (Xu and Brosche 2014), was up-regulated in active traps. Our findings suggest that ROS production is initiated by insect processing and that traps actively form a shield against detrimental ROS effects.

\section{Genome Research}

www.genome.org 
A

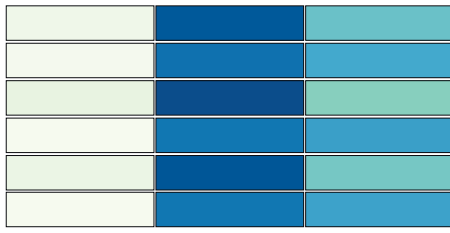

DGL1

LOX2

AOS

AOC3

OPR3

OPLC1

PPDA

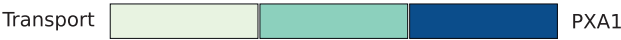

Oxidation

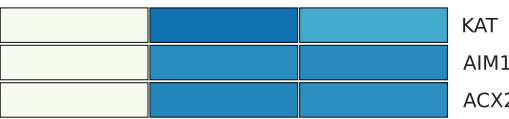

OPDA

$\downarrow$

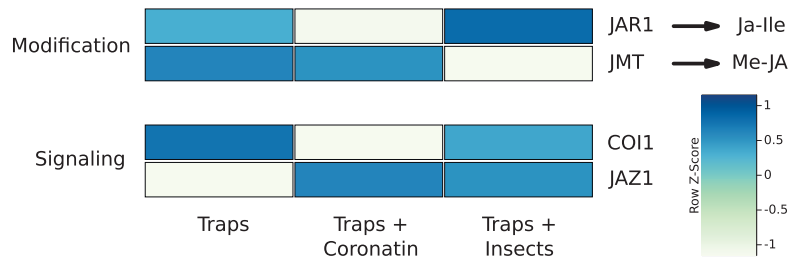

B

COI1

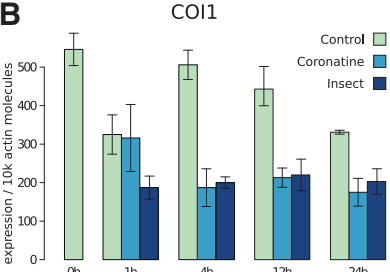

C $\quad J A Z 1$

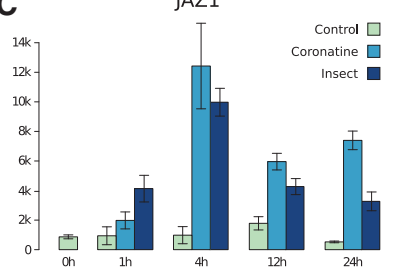

D VF CHITINASE I

SAG12

SCPL49
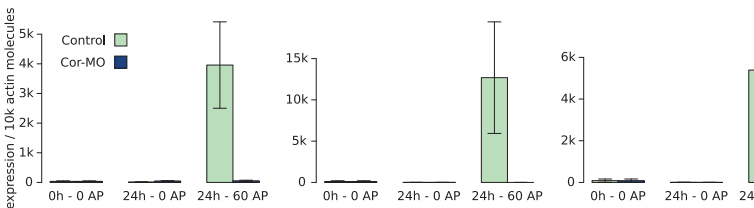

Figure 4. Activated Venus flytraps show signs of elevated jasmonate signaling. (A) Heat map depicting the expression of key genes mediating jasmonate biosynthesis, transport, and signaling in activated (COR/insect) versus nonstimulated traps. Expression values are scaled by rows (Z-scoring). The majority of the components are transcriptionally activated by insect or COR stimulation in traps and glandular tissue. Genes encoding the key enzymes of jasmonic acid $(J A)$ biosynthesis such as LOX2 (LIPOXYGENASE 2), AOS (ALLENEOXIDE SYNTHASE), and OPR3 (OXOPHYTODIENOATE-REDUCTASE 3) were highly induced in insectand COR-stimulated traps and glands. Likewise, the ABC-transporter PXA1 (PEROXISOMAL ABC-TRANSPORTER 1) mediating the import of the IA precursor OPDA (12-oxo-phytodioneic acid) into peroxisomes, and peroxisomal enzymes of the beta-oxidation chain generating JA from OPDA were stimulus induced. JAR1 (JASMONIC ACID RESISTANT 1), which finally converts IA into its physiologically active form JA-lle, appears specifically induced by insects. $(B, C)$ Quantitative PCR (qPCR) of the Dionaea JA receptor COI1 (CORONATINE INSENSITIVE 1) and its coreceptor/repressor JAZ1 (JASMONATE-ZIM-DOMAIN 1). (D) Effect of the JA-antagonist coronatine-O-methyloxime (COR-MO) on electro-mechanical induction of hydrolase expression. Traps were pretreated $4 \mathrm{~h}$ before application of zero or 60 APs with $\mathrm{H}_{2} \mathrm{O}$ (green) or $100 \mu \mathrm{M}$ COR-MO (blue). Transcript numbers are given relative to 10,000 molecules of DmACT1 \pm $\mathrm{SE}, n=6$. RNA was sampled after $24 \mathrm{~h}$ in response to zero or 60 recorded action potentials (APs). qPCR Abbreviations are as follows: (DGL) DONGLE PLA1-type phospholipase; (LOX2) LIPOXYGENASE 2; (AOS) ALLENE OXIDE SYNTHASE; (AOC3) ALLENE OXIDE CYCLASE 3; (OPR3) 12 OXOPHYTODIENOATE REDUCTASE 3; (OPCL1) OPC-8:0 COA LIGASE1; (PXA1) PEROXISOMAL ABC TRANSPORTER 1; (KAT) PEROXISOMAL 3KETOACYL-COA THIOLASE 3; (AIM1) ABNORMAL INFLORESCENCE MERISTEM; (ACX2) ACYL-COA OXIDASE 2; (JAR1) JASMONATE RESISTANT 1; and (JMT) JASMONIC ACID CARBOXYL METHYLTRANSFERASE (Dave and Graham 2012).

\section{Accessing prey nutrients}

When commencing digestion of the victim, metabolites and minerals containing the essential plant nutrients nitrogen $(\mathrm{N})$, phosphorus $(\mathrm{P})$, potassium $(\mathrm{K})$, calcium $(\mathrm{Ca})$, sulfur $(\mathrm{S})$, and magnesium (Mg) are released (Adamec 1997). Absorption of the products of digestion is mediated by glands aided by stimulus-induced formation of cuticular gaps (Robins and Juniper 1980; Joel et al. 1983). Accordingly, we observed that upon insect stimulation of traps or glands and the increasing availability of prey-derived nutrients, the expression profile of the Dionaea transportome changes dramatically. We identified 145 down-regulated and 148 upregulated transcripts, which are classified as transporters according to the transporter classification (TC) system (Supplemental Table S15). By using all TC-database classified transcripts as background, we sought for the most significantly enriched class. As would be expected from a nutrient uptake system, secondary active-transporters represented the most significantly enriched class (GAGE $q$-value: $2.6 \times 10^{-13}$ ). Regarding the subcellular localization of the corresponding transport proteins, we observed that the plasma membrane transporters were most strongly regulated (GAGE $q$-value: $3.5 \times 10^{-8}$ ) within all differentially expressed transporters. On the contrary, plastid localized transporters (GAGE $q$-value: $1.3 \times$ $10^{-9}$ ) appeared down-regulated, with the exception of the energy-supplying, plastidic ATP/ADP antiporter NTT1, which was strongly induced (Flügge et al. 2011).

Focusing on the substrate classes of the transporters, we found metal, nitrogen, and cation transporters to be the most regulated classes (Supplemental Table S16). We identified the highly induced plasma membrane phosphate transporters PHT1 and PHT2 (Shin et al. 2004). Consistent with the essential requirement of the molybdenum (Mo) cofactor in catalytic centers of several plant metabolic key enzymes, including ABA synthesis, a high-affinity molybdate transporter MOT1 (Tomatsu et al. 2007) was prominent in insect processing glands. Likewise, plasma membrane transporters for sulfate, as well as nitrogen-containing solutes, were induced by insects, suggesting that metabolites and macromolecules, such as amino acids, peptides, and nucleotides/ DNA, all represent part of Dionaea's diet. Furthermore, we found that the Dionaea ammonium transporter DmAMT1 is induced in active traps, as well as a Dionaea AKT1-like potassium channel and a HAK5-like high-affinity potassium transporter (Scherzer et al. 2015). When we analyzed the kinetics of transporter expression, it became apparent that upon trap stimulation, the expression of DmAMT1, DmHAK5, and DmHKT1 was induced 2-4 h after the onset of the stimulus, reaching peak expression after $12 \mathrm{~h}$ (Fig. 5A).

To test how the increased RNA abundance of DmAMT1, DmHAK5, and DmHKT1 translates into transporter activity, we stimulated Dionaea traps with COR and followed the time course of membrane potential changes of glands in response to $\mathrm{NH}_{4}^{+}, \mathrm{K}^{+}$, and $\mathrm{Na}^{+}$feeding. Comparing the transporter transcription and gland plasma membrane depolarization in response to the cations tested, we found that transporter activity lagged $>3 \mathrm{~h}$ behind gene expression (Fig. 5A). Detailed analysis using qPCR demonstrated that frequent firing of APs strongly triggered the expression of transporters involved in nutrient acquisition (Fig. 5B). As observed for hydrolases (see Fig. 4D), their transcriptional induction is inhibited upon blocking JA signaling downstream from APs by pretreating traps with the recently identified JAantagonist COR-MO (Fig. 5B; Monte et al. 2014). Taken together, our findings suggest that stimulated glands translate hapto- 
A
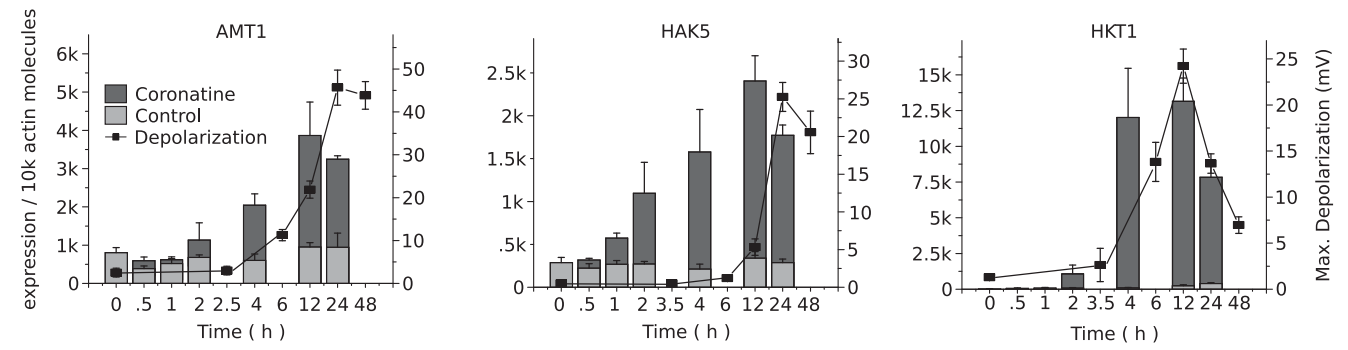

B
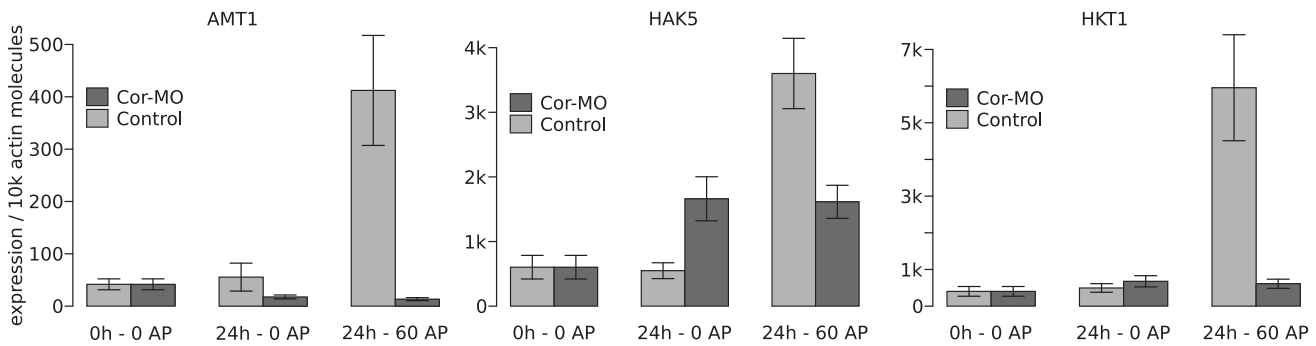

Figure 5. Cation transporters are highly up-regulated in active traps. (A) Coronatine-induced expression kinetics based on qPCR data of ammonium (DmAMT1; left), potassium (DmHAK5; middle), and sodium (DmHKT1; right) transporters and time course of membrane potential depolarizations in gland cells in response to either $6 \mathrm{mM} \mathrm{NH}_{4}^{+}, 3 \mathrm{mM} \mathrm{K}^{+}$, or $12 \mathrm{mM} \mathrm{Na}^{+}$. (B) Effect of the JA-antagonist COR-MO on transcript levels of ammonium (DmAMT1; left), potassium (DmHAK5; middle), and sodium (DmHKT1; right) transporters in response to 60 elicited APs. Traps were pretreated with $\mathrm{H}_{2} \mathrm{O}$ (light gray) or 100 $\mu \mathrm{M}$ COR-MO (dark gray) for $4 \mathrm{~h}$ before imposition of APs. Transcript numbers are given relative to 10,000 molecules of $D m A C T 1 \pm S E, n=6$. Experimental conditions were as described for Figure 4D.

electric signaling into JA-dependent transcriptional regulation of hydrolases and transporters. This ensures perfect gearing of secretion and prey digestion to the acquisition of prey-derived nutrients.

\section{Active traps display conserved stress signals}

When noncarnivorous plants are wounded by herbivores, they produce the touch hormone JA. Upon JA binding to the receptor/coreceptor complex COI1-JAZ1, this hormone signaling induces a local as well as a systemic electrical signal (Mousavi et al. 2013; Hedrich et al. 2016). This results in the production of substances toxic to herbivores or in making the plant meal indigestible. To test the hypothesis that the overall transcriptomic response of insect-stimulated Dionaea traps resembles defined stress-related responses known from noncarnivorous plants, we performed an interspecies comparison of active Dionaea plants to known coexpressed gene clusters in A. thaliana (Zaag et al. 2015). Taking advantage of the Complete Arabidopsis Transcriptome MicroArray (CATMA) gene expression reference database for Arabidopsis, we identified a diverse pattern of validated abiotic and biotic stress-related gene clusters (Supplemental Table S17) up-regulated in active traps. Further exploration of this response based on available Arabidopsis microarray data did indeed show that according to semantic similarities of GO enrichments, the Dionaea transcriptomic profile during insect capture and digestion closely resembles that of plants facing herbivore attack or wounding as opposed to fungal or bacterial infections (Fig. 6).

\section{Discussion}

Darwin's pioneering work demonstrated the existence of carnivorous lifestyles in several plant genera (Darwin 1875). He became particularly fascinated with the snap traps of D. muscipula, which resemble a sensory motion system that operates at high speed and precision without nerves or muscles (Brownlee 2013). When Darwin exposed open flytraps with all kinds of nutrient sources ranging from meat pieces to nitrogen-rich chemicals (Darwin 1875), he realized that, over time, the capture organ slowly closes and starts secretion. From this phenomenon, he concluded that as well as a touch sensor, Dionaea operates a chemo-sensing system that assesses the quality of the food provided by the prey.

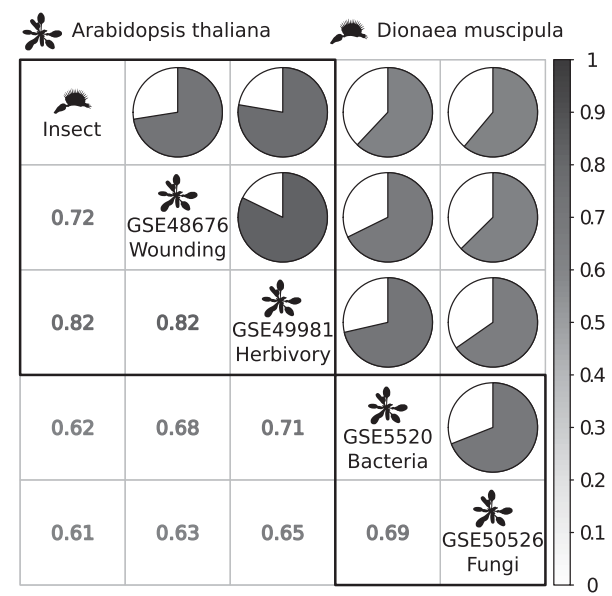

Figure 6. Prevailing signs of defense responses in active Dionaea traps. Semantic similarity between different Arabidopsis thaliana microarray experiments (GSE48676, GSE49981, GSE5520, and GSE50526) and active (insect-activated) traps. The semantic similarity is calculated by a quantitative comparison of all sets of significantly enriched gene ontology terms for each individual experiment. The lower triangle shows individual gene ontology similarities while the upper triangle visualizes the similarity as a relative pie chart. Black frames indicate results of the hierarchical clustering procedure using "complete" as agglomeration method. 
By taking advantage of the Venus flytrap as an experimental system that switches from a nonstimulated to an active insectprocessing state on demand, we provide unprecedented insights into the molecular and physiological processes that accompany plant carnivory. To obtain insights into the molecular processes underlying the Dionaea hunting cycle, we assembled a reference transcriptome using complementary sequencing data sets from inactive and active Dionaea. Rather than revealing specific anatomical and physiological properties, we found that, at the transcriptomic level, the nonstimulated Dionaea trap exhibits the expected hallmarks of any typical leaf (cf. petiole-trap overlap, Fig. 1C). What renders the trap transcriptome signature as different from the petiole is that nongreen glands share features with the root (Fig. 1D). Multicellular glands covering the inner surface of the trap represent the endocrine system of the trap and appear to be of tripartite functional morphology, organized in three layers. By combining results from EM ultrastructure with the gene expression patterns of glands at rest and under stimulation, we may assign a particular function to the cells in each of the three layers: (1) The inner layer 3 is densely packed with oil bodies, so it could serve as the energy reservoir for feeding the energy-dependent processes in the upper two layers; (2) layers 1 and 2 are engaged with the production and secretion of hydrolases that break down the prey trapped in the green stomach; and (3) prey processing and with some delay, the uptake of released nutrients very likely go hand-in-hand. The EM data suggest that the apical end of stimulated L1 cells is predominantly operating secretory vesicle fusion (Fig. 2; Supplemental Fig. S2). That the plasma membrane folding increases toward the basal end of these cells suggests that this cell pole of enlarged surface area is engaged, at least in part, with nutrient resorption (Adlassnig et al. 2012). Using the same reasoning, the stimulated cells in layer 2 , which are entirely folded in a brush-boarder-like fashion (Supplemental Fig. S2), would provide for gross nutrient uptake. Indeed, JA treatment of Arabidopsis has been shown to increase the membrane surface of metabolite shuttling transfer cells (Amiard et al. 2007; Adams et al. 2014), a situation resembling the ultrastructural changes toward the brushboarder-like plasma membrane folding observed in the stimulated gland cells of layer 2 .

We have shown that as well as mechano and JA hormone control of endocrine processes, chemo-sensing of the nutrient's nature provides for appraisal of the prey's presence and food quality. Our expression analyses showed that when chitin, a polymer of the insect exoskeleton, is supplied in addition to a mechanical stimulation, the expression of hydrolases, including VF CHITINASE I, increases a thousand-fold (Fig. 3C). This explains Darwin's observation and underlines the notion that for Dionaea, a living insect prey represents a mechanical as well as a chemical stimulus. What Darwin could not know at the time is that trigger hair stimulation, in other words mechanics, causes JA biosynthesis (Escalante-Perez et al. 2011; Böhm et al. 2016b). This is also the case for insect-stimulated traps of the carnivorous plant Drosera carpensis (Nakamura et al. 2013). JA not only represents a chemical trigger of gland cell secretion (Libiakova et al. 2014; Buch et al. 2015) but at the transcriptional level is able to partially substitute for insect action (see Figs. 3, 4A,B). In noncarnivorous plants, including Arabidopsis, JA biosynthesis is induced by herbivory and wounding (Gfeller et al. 2010).

Our transcriptomic studies demonstrate that active Dionaea traps operate stress-related pathways, including defense-associated responses (Supplemental Table S9). Included among the latter are hydrolytic enzymes secreted by carnivorous species, which are also transcriptionally induced upon pathogen challenge in noncarnivorous plants (Renner and Specht 2013). Although it has long been postulated that carnivory might have evolved from defense mechanisms, hitherto, there has been a lack of both molecular data and experimental approaches to separate nonstimulated from active trapping organs (Ibarra-Laclette et al. 2011, 2013; Leushkin et al. 2013; Barta et al. 2015). In this study, a comparative analysis of the transcriptomic profiles of nonstimulated versus active traps and glands demonstrates for the first time that, as well as hydrolases, a plethora of stress-associated pathways, including ROS and PCD signaling, are predominant in the activated Dionaea plant. This finding is further corroborated by an additional interspecies comparison that demonstrates that gene activities related to herbivory responses in noncarnivorous plants are prevalent in active traps (Fig. 6; Supplemental Table S17).

In response to insect attack, a traveling electrical wave translates into the rapid biosynthesis of JA (Mousavi et al. 2013) and its receptor-active derivative jasmonoyl-L-isoleucine (JA-Ile) (Campos et al. 2014). COI1-based perception of JA-Ile relieves JAZ proteinmediated negative transcriptional control, resulting in the production of secondary metabolites and proteins that exhibit well-known roles in defense. In the flytrap, a captured insect struggling to escape or COR treatment alone both activate the JA pathway (Fig. 4; Escalante-Perez et al. 2011; Böhm et al. 2016a). Activation of the JA pathway leads to the processing and consumption of, from the view of a noncarnivorous plant, a potential herbivore. Prey capture of the sundew Drosera induces both leaf bending and the accumulation of defense-related jasmonate phytohormones (Nakamura et al. 2013), and jasmonate increases the proteolytic activity in the pitcher fluid of the Nepenthes plant (Buch et al. 2015). Taken together, this suggests that Dionaea rewires defense responses known from noncarnivorous plants in order to operate its carnivorous lifestyle and that jasmonate signaling probably represents a basic mechanism in plant carnivory (Fig. 7).

In general, turning on defense is metabolically costly; it is frequently accompanied by significant growth inhibition and requires metabolic, transcriptional, and hormonal reprogramming (Yang et al. 2012; Campos et al. 2014). Initially, Dionaea invests energy in producing and growing traps and in providing the morphological and anatomical requirements for fast insect capture by storing elastic energy in a metastable configuration of the snap trap (Forterre et al. 2005). During this nonstimulated phase, glands are already producing hydrolases such as the Nepenthesin-like aspartic protease (Buch et al. 2015). Upon insect capture, this $\mathrm{pH}$ - and temperature-resistant broad range protease is probably among the first hydrolases released, with others being massively induced after chemical sensing and ongoing prey digestion. Insect-induced defense responses thus include hydrolase production, membrane remodeling, and secretion. In contrast to the typical defense response of noncarnivorous plants, the Dionaea trap actively suppresses cell death while fostering prey degradation and, more importantly, nutrient absorption. This is reflected in the expression of otherwise root-expressed transporters in stimulated glands. Little is known about the fate of prey-derived nutrients and their uptake, but by feeding isotope-labeled insects to traps, Kruse et al. (2014) showed that the organic nitrogen gained from insect prey was immediately redistributed and invested into the growth of new traps. Ammonium, an important nitrogenbased cation nutrient released from insect-derived glutamine by Dionaea deaminases (Scherzer et al. 2013), represents the substrate of the $\mathrm{NH}_{4}{ }^{+}$-selective uptake channel DmAMT1. As well as organic nutrients, degrading animal prey also releases potassium and 


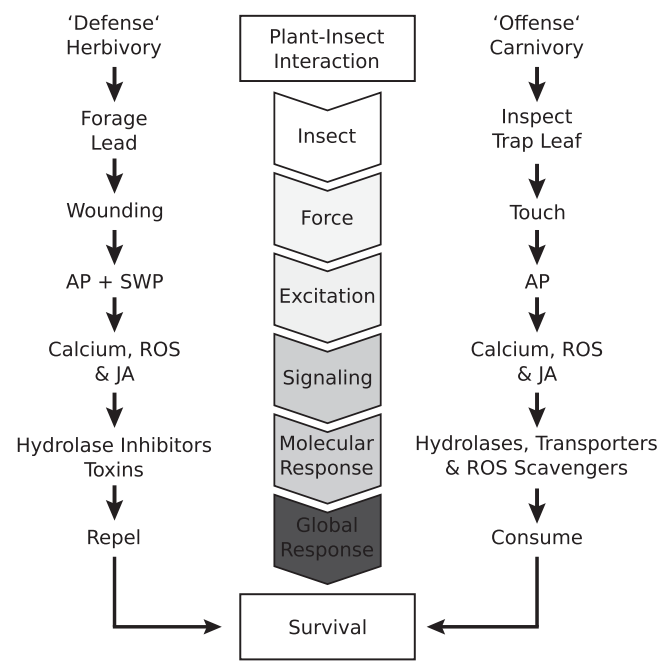

Figure 7. Turning defense into offense. Flow chart comparing the main events occurring at individual levels during the interaction of insects/herbivores with Dionaea or noncarnivorous plants. Similar to noncarnivorous plants, Dionaea attracts insects by means of volatile and nectar production (Kreuzwieser et al. 2014). Herbivores use plant leaves as a food source and by chewing, for example, caterpillars, impose a wounding event on the plant. In Dionaea, visiting insects generate touch events sensed by the trigger hairs of the trap leaf, triggering APs, while in noncarnivorous plants, slow-wave potentials (SWPs) or APs can be generated. APs represent traveling electrical waves that are capable of generating systemic responses in noncarnivores. In Dionaea, however, AP spreading is confined to the stimulated trap; it is not able to cross the trap-petiole anatomical barrier. Both wounding and touch-evoked electrical signaling trigger similar secondary signaling events: changes in cytosolic calcium concentration, production of reactive oxygen species (ROS), and synthesis of the touch hormone JA. Activation of JA signaling in noncarnivorous plants results in the production of a large number of specialized compounds with established roles in defense. This includes alkaloids, terpenoids, phenylpropanoids, phenolamides, amino acid derivatives, anti-nutritional proteins, and some pathogenesis-related (PR) proteins (Mithöfer and Boland 2012). Conversely, activation of the JA signaling pathway in Dionaea further leads to the expression of a broad spectrum of hydrolases (cf. Fig. 3), ROS scavengers, and finally nutrient uptake transporters. Thus, while in noncarnivorous plants the global objective is to repel a herbivore, Dionaea's only purpose is to consume it. Both strategies are costly, requiring the investment of a substantial amount of metabolic energy; if successful though, they significantly increase the chance of plant survival.

sodium. Dionaea requires potassium for turgor formation and trap reopening, as well as for growth and the development of new traps. For the uptake of prey-derived potassium ions, Dionaea glands operate two $\mathrm{K}^{+}$selective uptake systems in concert: a low-affinity, high-capacity $\mathrm{K}^{+}$channel DmAKT1 and a high-affinity, low-capacity proton-coupled $\mathrm{K}^{+}$transporter DmHAK5 (Scherzer et al. 2015). In Arabidopsis, HAK5 expression strongly depends on $\mathrm{K}^{+}$availability and is under control of the ERF/AP2-type transcription factor AtDDF2 (Hong et al. 2013). Expression of the Dionaea DDF2 is barely detectable in nonstimulated glands but is induced more than 150-fold and more than 400 -fold in glands stimulated by insects or COR, respectively (data not shown). Interestingly, active traps also take up the otherwise toxic $\mathrm{Na}^{+}$ions via a gland cell expressed $\mathrm{Na}^{+}$channel DmHKT1. Most likely, sodium ions are required for osmotic adjustment. In contrast to $\mathrm{K}^{+}$, however, $\mathrm{Na}^{+}$ accumulates only in mature traps (Böhm et al. 2016a) and is not channeled to young ones, presumably to protect young traps from its toxic effect during organ growth and development.

In summary, our work extends and refines the current model of the Dionaea hunting cycle. We show that the first mechano- electric stimulation of the trigger hair by a trap-visiting insect sets the trap into a "poised to capture" mode (Supplemental Fig. S3). One touch-induced AP is memorized by the trap but is insufficient for trap closure. A second AP elicited within a given period (about $\leq 20 \mathrm{sec}$ ) is required for fast closure and prey capture. When trying to escape, a prey insect repeatedly touches the mechano sensors, thereby eliciting the repetitive firing of APs. Two or more APs activate the JA signaling pathway. Upon five or more elicited APs, the capture organ becomes hermetically sealed and the victim is digested. Glands covering the inner surface of the stomach start to express genes encoding enzymes that decompose the prey into its nutrient building blocks, alongside the expression of transporters for the uptake of prey-derived nutrients. In the latter processes, mechano-electric stimulation can be substituted by direct JA hormone administration. The more often the trigger hairs are touched, APs are fired, and the touch hormone is synthesized, the longer and more active is the flytrap's endocrine system, a process further stimulated by "prey derived molecular patterns." By these means, the number of APs informs the plant about the size and nutrient content of the struggling prey.

In evolutionary terms, it seems remarkable that a generic wounding response pathway that exists in most plant species has been partly rewired to enable prey capture, digestion, and nutrient acquisition (Fig. 7). Following this strategy, the carnivorous syndrome not only covers expenses (keeping the trap active), but also gains a surplus, allowing the carnivorous plant to afford and grow new capture organs for future hunting cycles.

\section{Methods}

\section{Plant growth}

Dionaea plants were grown as described previously (Scherzer et al. 2013). Briefly, D. muscipula plants were purchased from CRESCO Carnivora and grown in plastic pots at $22^{\circ} \mathrm{C}$ in a 16 -h:8-h lightdark photoperiod. All experiments were performed with healthy mature plants. Nonstimulated traps, petioles, roots, trap rims, and flowers were immediately frozen in liquid nitrogen and processed. Traps and glands were stimulated by feeding traps with live crickets or by spraying a $100 \mu \mathrm{M}$ COR solution (SigmaAldrich) directly onto open traps. Tissues were harvested upon trap closure or secretion onset (within 4-12 h) and immediately frozen in liquid nitrogen. Isolation of secretory gland complexes was achieved by gently abrading the inner trap surface using a sharp razor blade. For mechanical induction of gene expression, trigger hairs were stimulated 60 times $(1 / \mathrm{min})$, and samples were collected $24 \mathrm{~h}$ after the first stimulus. In inhibitor tests, $100 \mu \mathrm{M}$ COR-MO (synthesized according to the method described by Böhm et al. 2016b) was sprayed $4 \mathrm{~h}$ before mechanical stimulation was applied to the Venus flytraps.

\section{RNA extraction, sequencing, and qPCR}

RNA was isolated separately from each sample using a modified cetyltrimethylammonium bromide (CTAB)-based protocol. In brief, $0.1 \mathrm{~g}$ plant material powdered in liquid nitrogen was thoroughly mixed with $0.7 \mathrm{~mL}$ of hot $\left(65^{\circ} \mathrm{C}\right)$ RNA-extraction buffer (2\% CTAB, 2\% polyvinylpyrrolidone K 25 [PVP], $100 \mathrm{mM}$ TRIS/ $\mathrm{HCl}$ at $\mathrm{pH} 8.0,25 \mathrm{mM} \mathrm{Na}-E D T A$ at $\mathrm{pH} 8.0,2 \mathrm{M} \mathrm{NaCl}$, with $2.5 \%$ [v/v] 2-mercaptoethanol added immediately before use). Following $10 \mathrm{~min}$ incubation at $65^{\circ} \mathrm{C}$ and extraction with 1 vol of chloroform/isoamyl alcohol $(24: 1, \mathrm{v} / \mathrm{v})$, RNA was precipitated from the supernatant by adding $175 \mu \mathrm{L}$ of $8 \mathrm{M} \mathrm{LiCl}$ overnight $\left(4^{\circ} \mathrm{C}\right)$. RNA was collected by centrifugation, resuspended in

\section{Genome Research}

www.genome.org 
DEPC $\mathrm{H}_{2} \mathrm{O}$, and precipitated in the presence of $0.1 \mathrm{vol} 3 \mathrm{M} \mathrm{Na}$ acetate (pH 5.2) and $2.5 \mathrm{vol}$ of $96 \%$ EtOH. After a washing step using $70 \% \mathrm{EtOH}$, RNA was dissolved in $40 \mu \mathrm{L}$ of DEPC $\mathrm{H}_{2} \mathrm{O}$. DNA contamination was removed by DNase I treatment on a column (Roche). RNA quantity and quality were determined by capillary electrophoresis (Experion automated electrophoresis system and Experion RNA high sense analysis kit, Bio-Rad Laboratories). TruSeq RNA libraries (partially stranded) were generated, and RNA sequencing was performed using an Illumina HiSeq 2000 sequencer at LGC Genomics.

Individual transcript levels were analyzed by quantitative real-time PCR (qPCR). qPCR was performed using a Realplex Mastercycler (Eppendorf), 1:20 diluted cDNA, and the ABsolute QPCR SYBR green capillary mix (Thermo Scientific). Expression levels were quantified using a standard for each primer pair and normalized to 10,000 molecules of actin (DmACT) cDNA transcripts.

Primers employed were as follows: SCPL49LCfwd, 5'-AC TTAATCCGGGTATCA-3'; SCPL49LCrev， 5'-AGGTCCATAGGTA TTCA-3'; VF CHITINASE ILCfwd, 5'-GAAAGTTATTACGGTCG3'; VF CHITINASE ILCrev, 5'-CTTTACCACACTCAACG-3'; SAG 12fwd, 5'-CGCATTCGAGTATATGA-3'; SAG12rev, 5'-CAACATT CCTTTGCATC-3'; AMT1LCfwd, 5'-TTGCT ACCAAGAAACAC-3'; AMT1LCrev, 5'-TGAGTTGATGTAAGGAG-3'; HAK5.1LCfwd, 5'GTATGTTT TGTGCTGG-3'; HAK5.1Lrev, 5'-GTCCGAGCATATAA GTC-3'; HKT1LCfw, 5'-GAATGTAGCAGT CGAG-3'; HKT1LCrev, 5'-CCCGTGCATGTTAAAT-3'; JAZ1.1LCfwd， 5'-GTGTTCAACGA CTTCC-3'; JAZ1.1LCrev, 5'-TTGTTAAGGTGTATGGC-3'; COI1L Cfwd, 5'-TTACGTCGGACTGTAT-3'; and COI1LCrev, 5'-AACTCT AAGCTAAGACAT-3'.

\section{Data preparation and transcriptome assembly}

Raw read data sets were screened for quality issues with FastQC (version 0.11.4). Passed data sets were quality trimmed using skewer (version 0.1.67; -Q 30; -q 30; -l 75; -m pe) (Jiang et al. 2014) The transcriptome was assembled using Trinity (release 2013-02-16; -jaccard_clip; -min_kmer_cov 2; -path_reinforcement_distance 75) (Grabherr et al. 2011). The assembly was screened for artificial fusion events caused by low-complexity regions or highly similar UTRs. A linkage map was constructed for transcripts with more than one potential coding region (see below). Evidence from homology-based database searches and mapped paired-end reads were used to link the potential coding regions. Paired-end mappings were produced with Bowtie (version 0.12.7) (Langmead et al. 2009), while homology evidence was generated by searching transcripts against a plantcomprising subset of the UniProt database (2015; release 2014-0619) (UniProt-Consortium 2015) using BLAST (version 2.2.29) (Camacho et al. 2009). Where no linkage evidence was found, the transcript was considered to be fused. Fusion sites were detected by searching for the lowermost covered site between two coding regions. Transcripts were cut apart, and the region with the lowermost coverage was trimmed from both the resulting transcripts. Whenever a transcript was defused, the original isoform-gene relation returned by Trinity was disregarded. To recreate a reliable isoform-gene relation, we partitioned all defused isoforms derived from the same gene using transitivity clustering (version 1.0) (Wittkop et al. 2010) into new genes. If sufficient evidence for a linkage was found, the transcripts were considered intron containing. Introns were removed by aligning high-scoring templates from the homology search to the transcript using GeneWise (version 2.4.1) (Birney et al.2004). Only proper alignments were used to cut introns from transcripts; otherwise, transcripts were left untouched.

\section{Feature annotation}

Refined transcripts were annotated using homology- and profile-based methods. Coding regions were identified using TransDecoder (http://transdecoder.github.io; release 2014-0116). Putative peptides were preferred when they had a significant match to a Pfam domain. Only peptides with a length of at least 30 aa were considered. Protein families and domains were classified using InterProScan (release 44.0) Quevillon et al. 2005). Phobius (version 1.01) (Kall et al. 2004), SignalP (version 4.0) (Petersen et al. 2011), and TMHMM (version 2.0) (Moller et al. 2001) were integrated into the default signature recognition methods. GO terms were predicted using Blast2GO (version 2.5.2) (Conesa et al. 2005). GO terms were augmented with Interpro annotations using ANNEX (Myhre et al. 2006). MapMan bins were annotated with Mercator (Lohse et al. 2014). Interspersed repeats and low-complexity regions were identified using RepeatMasker (version 4.0.3) (Smit et al. 2013-2015). Putative orthologs between the Venus flytrap transcriptome and A. thaliana ("TAIR10") (Lamesch et al. 2012) were assigned using a conservative conditional reciprocal best BLAST (CRB-BLAST; release 2015-05-19) (Aubry et al. 2014). TCDB classifications were assigned with a two-step approach. First, a CRB-BLAST against the TCDB sequence set was used to identify conditional reciprocal best hits. If no distinct one-to-one hit was found, the sum of the best hits ( $e$-value $\leq 10^{-5}$ ) was evaluated. If all hits were annotated with the same TCDB family, the annotation was mapped to the Venus flytrap sequence.

\section{Transcript filtering}

Prior to differential expression analyses and enrichment studies, the following filtering steps were applied to the refined assembly: (1) Noncoding RNAs were excluded by searching for isoforms without a potential coding region; (2) ambiguous low abundance genes were excluded when no sequenced sample produced an expected count higher than five; (3) in addition, ambiguous isoforms were removed by only considering isoforms with an abundance $>1 \%$ of the abundance of its parental gene; and (4) possible contaminations were filtered with a two-step approach. Isoforms were split into overlapping $k$-mers ( $k$-mer size $19 \mathrm{bp}$ ). Each $k$-mer was then searched in a database of trusted $k$-mers created from genomic sequencing data (data not shown). Isoforms without a single $k$-mer present in the trusted $k$-mer databases were disregarded. The remaining isoforms were searched against the complete nonredundant database (release 2015-01-13) (Sayers et al. 2009) using BLASTN. Resulting hits were taken to calculate the lowest common ancestor (LCA) with MEGAN4 (Huson et al. 2011), using default settings. Isoforms with a LCA in bacteria, fungi, or metazoa were disregarded. (5) Transposon-like isoforms detected by RepeatMasker were excluded if they only contained the transposable element or a protein domain associated with interspersed repeats in the current RepBase release (release 2014-04-2) (Jurka et al. 2005).

\section{Transcript abundance estimation and differential expression testing}

Isoform and gene abundances were quantified with RSEM (version 1.2.5) (Li and Dewey 2011) using the unfiltered read data sets. Differential expression tests and enrichment analysis, as well as abundance visualizations, were carried out using gene counts. Isoform abundances were only used to select a representing isoform for genes of interest. The manual selection prioritized isoforms over others that were complete (presence of ORF with flanking UTRs), and had a protein domain annotation and an 
expression profile similar to its parental gene. Gene count matrices for each experiment were normalized using the normalization method implemented in the DESeq package (version 1.22.0) (Anders and Huber 2010). Samples that were possible outliers were detected using arrayQualityMetrics (version 3.26.0) (Kauffmann et al. 2009) and a variance stabilized transformation of the normalized gene count data set. The same expression data set was used for the PCA. DEGs were detected using DESeq. Only genes exhibiting an adjusted $P$-value equal or smaller than 0.01 were considered as significant. DEGs for $A$. thaliana microarray experiments (GSE48676, GSE49981, GSE5520, and GSE50526) were detected using GEO2R (Barrett et al. 2013).

\section{Enrichment analysis and semantic similarity}

GO and GOSlim enrichment studies were carried out using topGO (version 2.22.0) (Alexa et al. 2006) using the generic GO and the plant slim subset developed by The Arabidopsis Information Resource ("TAIR"). Measures for differential expression ( $P$-value) were included using the weighted algorithm, while Fisher's exact test was used as a test statistic. Terms were considered significant with a $P$-value equal or smaller than 0.01 after Bonferroni correction. Semantic similarity of GO enrichments was measured with the GNU R Bioconductor package GOSemSim (Yu et al. 2010) using Wang's measurement method and the best-match average (BMA) strategy to combine scores for individual terms. Gene set enrichments (GSEAs) were carried out with GAGE (Luo et al. 2009) using MapMap bins, TCs, or manually assigned gene classifications. DESeq derived adjusted $P$-values were used as per gene score, and gene sets were considered significant at a $q$-value equal or lower than 0.1 .

\section{Definition of the putative secretome and transportome}

The secretome of the Venus flytrap was defined with the following rule set. Each putative member had to be a DEG when comparing nonstimulated with insect-stimulated glands (both down- and upregulated DEGs were considered). The underlying gene needed to have at least one differentially expressed isoform with an annotated signal peptide and evidence of a detectable peptide in the same open reading frame. The transportome was defined similar to the secretome. Putative members had to be differentially expressed in nonstimulated or insect-stimulated glands. The gene needed to have at least one differentially expressed isoform annotated with two or more transmembrane domains. Furthermore, the same sequence needed to have a proper TCDB classification as described above.

\section{Proteomics}

\section{Protein preparation for mass spectrometry}

Sampling of secreted fluid and gel-free proteomics of the digestive fluid was performed essentially as described in Schulze et al. (2012). A total of $50 \mu \mathrm{g}$ protein was predigested for $3 \mathrm{~h}$ with endoproteinase Lys-C ( $0.5 \mu \mathrm{g} / \mu \mathrm{L}$; Wako Chemicals) at room temperature (RT). After fourfold dilution with $10 \mathrm{mM}$ Tris- $\mathrm{HCl}(\mathrm{pH} \mathrm{8)}$, samples were digested with $4 \mu \mathrm{L}$ sequencing grade modified tryp$\sin \left(0.5 \mu \mathrm{g} \mathrm{L}^{-1}\right.$; Promega) overnight at $37^{\circ} \mathrm{C}$. After overnight digestion, trifluoroacetic acid (TFA) was added (until $\mathrm{pH} \leq 3$ ) to stop digestion. Digested peptides were desalted over C18 tips (Rappsilber et al. 2003).

\section{LC-MS/MS analysis of peptides and phosphopeptides}

Tryptic peptide mixtures were analyzed by LC/MS/MS using nanoflow easy-nLC1000 (Thermo Scientific) as an HPLC-system and a quadrupole-orbitrap hybrid mass spectrometer (Q-Exactive Plus, Thermo Scientific) as a mass analyzer. Peptides were eluted from a $75-\mu \mathrm{m} \times 50-\mathrm{cm}$ C18 analytical column (PepMan, Thermo Scientific) on a linear gradient running from $4 \%$ to $64 \%$ acetonitrile in $120 \mathrm{~min}$ and sprayed directly into the Q-Exactive mass spectrometer. Proteins were identified by MS/MS using information-dependent acquisition of fragmentation spectra of multiple charged peptides. Up to 12 data-dependent MS/MS spectra were acquired for each full-scan spectrum acquired at 70,000 full-width half-maximum resolution. Fragment spectra were acquired at a resolution of 35,000 . The overall cycle time was $\sim 1$ sec.

Protein identification and ion intensity quantitation was carried out by MaxQuant version 1.5.3.8 (Cox and Mann 2008). Spectra were matched against the Dionaea contigs using Andromeda (Cox et al. 2011). Carbamidomethylation of cysteine was set as a fixed modification, and oxidation of methionine was set as variable modification. Mass tolerance for the database search was set to $20 \mathrm{ppm}$ on full scans and 0.5 Da for fragment ions. Multiplicity was set to one. For label-free quantitation, retention time matching between runs was chosen within a time window of $2 \mathrm{~min}$. The peptide false-discovery rate (FDR) and protein FDR were set to 0.01, while site FDR was set to 0.05. Hits to contaminants (e.g., keratins) and reverse hits identified by MaxQuant were excluded from further analysis.

\section{Mass spectrometric data analysis and statistics}

Reported ion intensity values were used for quantitative data analysis. cRacker (Zauber and Schulze 2012) was used for label-free data analysis based on the MaxQuant output (evidence.txt). All phosphopeptides and proteotypic nonphosphopeptides were used for quantitation. Within each sample, ion intensities of each peptide ion species (each $\mathrm{m} / \mathrm{z}$ ) were normalized against the total ion intensities in that sample (peptide ion intensity/total sum of ion intensities). Subsequently, each peptide ion species (i.e., each $\mathrm{m} / \mathrm{z}$ value) was scaled against the average normalized intensities of that ion across all treatments. For each peptide, values from three biological replicates then were averaged after normalization and scaling.

\section{Electron microscopy}

Plant material was cut into small pieces and immediately fixed in $5 \%$ glutaraldehyde in a $0.1 \mathrm{M}$ cacodylate buffer for $3 \mathrm{~h}$ at $4^{\circ} \mathrm{C}$. Pieces were washed twice, first with $7 \%$ sucrose in a $0.1 \mathrm{M}$ cacodylate buffer and then with a $0.1 \mathrm{M}$ cacodylate buffer alone. For postfixation, the solution was replaced by $2 \%$ osmium tetroxide in a $0.1 \mathrm{M}$ cacodylate buffer for $2 \mathrm{~h}$ on ice. Following a three-stage washing step with $0.05 \mathrm{M}$ cacodylate buffer, the plant material was dehydrated using an ascending ethanol series and then stored in 100\% ethanol overnight at RT. After two 1-h incubations at RT in $100 \%$ ethanol and propylene oxide, respectively, the plant material was again stored overnight in fresh propylene oxide at RT. The next day, it was equilibrated with and subsequently embedded in Spurr's epoxy resin (Spurr 1969). After changing the solution several times using an increasing ratio of Spurr:propylene oxide, plant pieces were transferred to appropriate embedding molds filled with $100 \%$ Spurr. Contrasted ultrathin sections $(60-70 \mathrm{~nm})$ of the embedded plant material were examined using a JEOL JEM-2100 TEM.

\section{Electrophysiology}

Prior to measurements, the lobe of a cut trap was glued to the bottom of a recording chamber and left to recover for $30 \mathrm{~min}$ in a standard solution containing $0.1 \mathrm{mM} \mathrm{KCl}, 10 \mathrm{mM} \mathrm{CaCl}_{2}$, and $15 \mathrm{mM}$ MES/TRIS ( $\mathrm{pH}$ 6). Osmolarity was adjusted to $240 \mathrm{mOsm} / \mathrm{kg}$ using

\section{Genome Research}

www.genome.org 
D-sorbitol. Membrane potential recordings were performed as described elsewhere (Scherzer et al. 2015) using a standard solution supplemented with varying concentrations of either $\mathrm{NaCl}, \mathrm{KCl}$, or $\mathrm{NH}_{4} \mathrm{Cl}$ as indicated in the figure legend.

\section{Data access}

The sequence data from this study have been submitted to the NCBI BioProject (http://www.ncbi.nlm.nih.gov/bioproject) under accession number PRJNA203407. Sequence annotations, expression quantifications, and differential testing results of the Venus flytrap reference transcriptome are available in the Supplemental Tables S1-S17 and through the Carnivorome transcriptome browser (http://tbro.carnivorom.com). The mass spectrometry proteomics data have been submitted to the ProteomeXchange Consortium (http://www.proteomexchange.org) via the PRIDE partner repository (http://www.ebi.ac.uk/pride/archive/) (Vizcaino et al. 2016) with the data set identifier PXD003480.

\section{Acknowledgments}

We thank T.A. Cuin and I. Marten for helpful discussion and critical reading of the manuscript. We thank B. Neumann for excellent technical assistance. We thank G. Krohne for technical expertise in high-quality REM imaging. This work was supported by the European Research Council under the European Union's Seventh Framework Program (FP/20010-2015)/ERC Grant Agreement no. 250194-Carnivorom and Deanship of Scientific Research, King Saud University, International Research Group Program (IRG14-08), to R.H. and K.A.A.-R. F.B. and M.A. were supported by a grant of the German Excellence Initiative to the Graduate School of Life Sciences, University of Würzburg.

Author contributions: R.H., K.A.A.-R., I.K., and D.B. conceived the work. F.B., J.S., M.A., A.L.V.d.W., I.K., D.B., and R.H. designed experiments and performed bioinformatic data analyses. W.X.S. conducted proteomic experiments; E.K., electrophysiological experiments. C.L. and D.B. analyzed cell biology data sets. M.E.P. and I.K. carried out molecular biology experiments. R.H., D.B., F.B., J.S., I.K., A.P.W., and A.M. wrote the manuscript.

\section{References}

Adamec L. 1997. Mineral nutrition of carnivorous plants: a review. Bot Rev 63: $273-299$.

Adams WW III, Cohu CM, Amiard V, Demmig-Adams B. 2014. Associations between the acclimation of phloem-cell wall ingrowths in minor veins and maximal photosynthesis rate. Front Plant Sci 5: 24.

Adlassnig W, Koller-Peroutka M, Bauer S, Koshkin E, Lendl T, Lichtscheidl IK. 2012. Endocytotic uptake of nutrients in carnivorous plants. Plant J 71: 303-313.

Albert V, Williams S, Chase M. 1992. Carnivorous plants: phylogeny and structural evolution. Science 257: 1491-1495.

Alexa A, Rahnenfuhrer J, Lengauer T. 2006. Improved scoring of functional groups from gene expression data by decorrelating GO graph structure. Bioinformatics 22: 1600-1607.

Amiard V, Demmig-Adams B, Mueh KE, Turgeon R, Combs AF, Adams WW III. 2007. Role of light and jasmonic acid signaling in regulating foliar phloem cell wall ingrowth development. New Phytol 173: 722-731.

Anders S, Huber W. 2010. Differential expression analysis for sequence count data. Genome Biol 11: R106.

Antolin-Llovera M, Ried MK, Binder A, Parniske M. 2012. Receptor kinase signaling pathways in plant-microbe interactions. Annu Rev Phytopathol 50: 451-473.

Aubry S, Kelly S, Kumpers BM, Smith-Unna RD, Hibberd JM. 2014. Deep evolutionary comparison of gene expression identifies parallel recruitment of trans-factors in two independent origins of $\mathrm{C}_{4}$ photosynthesis. PLoS Genet 10: e1004365.

Barrett T, Wilhite SE, Ledoux P, Evangelista C, Kim IF, Tomashevsky M, Marshall KA, Phillippy KH, Sherman PM, Holko M, et al. 2013. NCBI
GEO: archive for functional genomics data sets: update. Nucleic Acids Res 41: D991-D995.

Barta J, Stone JD, Pech J, Sirova D, Adamec L, Campbell MA, Storchova H. 2015. The transcriptome of Utricularia vulgaris, a rootless plant with minimalist genome, reveals extreme alternative splicing and only moderate sequence similarity with Utricularia gibba. BMC Plant Biol 15: 78.

Birney E, Clamp M, Durbin R. 2004. GeneWise and Genomewise. Genome Res 14: 988-995.

Böhm J, Scherzer S, Shabala S, Krol E, Neher E, Mueller TD, Hedrich R. 2016a. Venus flytrap HKT1-type channel provides for prey sodium uptake into carnivorous plant without conflicting with electrical excitability. Mol Plant 9: 428-436.

Böhm J, Scherzer S, Krol E, Kreuzer I, von Meyer K, Lorey C, Mueller TD, Shabala L, Monte I, Solano R, et al. 2016b. The Venus flytrap Dionaea muscipula counts prey-induced action potentials to induce sodium uptake. Curr Biol 26: 286-295.

Brownlee C. 2013. Carnivorous plants: trapping, digesting and absorbing all in one. Curr Biol 23: R714-R716.

Bruggeman Q, Raynaud C, Benhamed M, Delarue M. 2015. To die or not to die? Lessons from lesion mimic mutants. Front Plant Sci 6: 24.

Buch F, Kaman WE, Bikker FJ, Yilamujiang A, Mithofer A. 2015. Nepenthesin protease activity indicates digestive fluid dynamics in carnivorous nepenthes plants. PLoS One 10: e0118853.

Burdon-Sanderson J. 1872. Note on the electrical phenomena which accompany irritation of the leaf of Dionaea muscipula (Venus flytrap). Proc R Soc 21: 495-496.

Camacho C, Coulouris G, Avagyan V, Ma N, Papadopoulos J, Bealer K, Madden TL. 2009. BLAST+: architecture and applications. BMC Bioinformatics 10: 421.

Cameron KM, Wurdack KJ, Jobson RW. 2002. Molecular evidence for the common origin of snap-traps among carnivorous plants. Am J Bot 89: 1503-1509.

Campos ML, Kang JH, Howe GA. 2014. Jasmonate-triggered plant immunity. J Chem Ecol 40: 657-675.

Cao HX, Schmutzer T, Scholz U, Pecinka A, Schubert I, Vu GT. 2015. Metatranscriptome analysis reveals host-microbiome interactions in traps of carnivorous Genlisea species. Front Microbiol 6: 526.

Carretero-Paulet L, Chang TH, Librado P, Ibarra-Laclette E, Herrera-Estrella L, Rozas J, Albert VA. 2015a. Genome-wide analysis of adaptive molecular evolution in the carnivorous plant Utricularia gibba. Genome Biol Evol 7: 444-456.

Carretero-Paulet L, Librado P, Chang TH, Ibarra-Laclette E, Herrera-Estrella L, Rozas J, Albert VA. 2015b. High gene family turnover rates and gene space adaptation in the compact genome of the carnivorous plant Utricularia gibba. Mol Biol Evol 32: 1284-1295.

Chini A, Fonseca S, Fernandez G, Adie B, Chico JM, Lorenzo O, GarciaCasado G, Lopez-Vidriero I, Lozano FM, Ponce MR, et al. 2007. The JAZ family of repressors is the missing link in jasmonate signalling. Nature 448: 666-671.

Conesa A, Gotz S, Garcia-Gomez JM, Terol J, Talon M, Robles M. 2005. Blast2GO: a universal tool for annotation, visualization and analysis in functional genomics research. Bioinformatics 21: 3674-3676.

Cox J, Mann M. 2008. MaxQuant enables high peptide identification rates, individualized p.p.b.-range mass accuracies and proteome-wide protein quantification. Nat Biotechnol 26: 1367-1372.

Cox J, Neuhauser N, Michalski A, Scheltema RA, Olsen JV, Mann M. 2011. Andromeda: a peptide search engine integrated into the MaxQuant environment. J Proteome Res 10: 1794-1805.

Darwin C. 1875. Insectivorous plants. Murray, London, UK.

Dave A, Graham IA. 2012. Oxylipin signaling: a distinct role for the jasmonic acid precursor cis-(+)-12-Oxo-phytodienoic acid (cis-OPDA). Front Plant Sci 3: 42.

Ellison AM, Gotelli NJ. 2009. Energetics and the evolution of carnivorous plants-Darwin's 'most wonderful plants in the world'. I Exp Bot 60: 19-42.

Escalante-Perez M, Krol E, Stange A, Geiger D, Al-Rasheid KA, Hause B, Neher E, Hedrich R. 2011. A special pair of phytohormones controls excitability, slow closure, and external stomach formation in the Venus flytrap. Proc Natl Acad Sci 108: 15492-15497.

Escalante-Perez M, Scherzer S, Al-Rasheid KA, Dottinger C, Neher E, Hedrich R. 2014. Mechano-stimulation triggers turgor changes associated with trap closure in the Darwin plant Dionaea muscipula. Mol Plant 7: 744-746.

Fleischmann A, Michael TP, Rivadavia F, Sousa A, Wang W, Temsch EM, Greilhuber J, Muller KF, Heubl G. 2014. Evolution of genome size and chromosome number in the carnivorous plant genus Genlisea (Lentibulariaceae), with a new estimate of the minimum genome size in angiosperms. Ann Bot 114: 1651-1663.

Flügge UI, Hausler RE, Ludewig F, Gierth M. 2011. The role of transporters in supplying energy to plant plastids. J Exp Bot 62: 2381-2392. 
Forterre Y, Skotheim JM, Dumais J, Mahadevan L. 2005. How the Venus flytrap snaps. Nature 433: 421-425.

Gfeller A, Liechti R, Farmer EE. 2010. Arabidopsis jasmonate signaling pathway. Sci Signal 3: $\mathrm{cm} 4$

Givnish TJ, Burkhardt EL, Happel RE, Weintraub JD. 1984. Carnivory in the bromeliad Brocchinia reducta, with a cost/benefit model for the general restriction of carnivorous plants to sunny, moist, nutrient-poor habitats. Am Nat 124: 479-497.

Grabherr MG, Haas BJ, Yassour M, Levin JZ, Thompson DA, Amit I, Adiconis X, Fan L, Raychowdhury R, Zeng Q, et al. 2011. Full-length transcriptome assembly from RNA-Seq data without a reference genome. Nat Biotechnol 29: 644-652.

Hedrich R, Salvador-Recatala V, Dreyer I. 2016. Electrical wiring and longdistance plant communication. Trends Plant Sci pii: S1360-1385(16) 00032-7.

Heubl G, Bringmann G, Meimberg H. 2006. Molecular phylogeny and character evolution of carnivorous plant families in Caryophyllales: revisited. Plant Biol (Stuttg) 8: 821-830.

Hong J-P, Takeshi Y, Kondou Y, Schachtman DP, Matsui M, Shin R. 2013. Identification and characterization of transcription factors regulating Arabidopsis HAK5. Plant Cell Physiol 54: 1478-1490.

Howell SH. 2013. Endoplasmic reticulum stress responses in plants. Annu Rev Plant Biol 64: 477-499.

Huson DH, Mitra S, Ruscheweyh HJ, Weber N, Schuster SC. 2011. Integrative analysis of environmental sequences using MEGAN4. Genome Res 21: 1552-1560.

Ibarra-Laclette E, Albert VA, Perez-Torres CA, Zamudio-Hernandez F, OrtegaEstrada Mde J, Herrera-Estrella A, Herrera-Estrella L. 2011. Transcriptomics and molecular evolutionary rate analysis of the bladderwort (Utricularia), a carnivorous plant with a minimal genome. BMC Plant Biol 11: 101.

Ibarra-Laclette E, Lyons E, Hernandez-Guzman G, Perez-Torres CA, Carretero-Paulet L, Chang TH, Lan T, Welch AJ, Juarez MJ, Simpson J, et al. 2013. Architecture and evolution of a minute plant genome. Nature 498: 94-98.

Jiang H, Lei R, Ding SW, Zhu S. 2014. Skewer: a fast and accurate adapter trimmer for next-generation sequencing paired-end reads. $B M C$ Bioinformatics 15: 182 .

Joel DM, Rea PA, Juniper BE. 1983. The cuticle of Dionaea-muscipula Ellis (Venus flytrap) in relation to stimulation, secretion and absorption. Protoplasma 114: $44-51$

Juniper BE, Robins RJ, Joel DM. 1989. The carnivorous plants. Academic Press, New York.

Jurka J, Kapitonov VV, Pavlicek A, Klonowski P, Kohany O, Walichiewicz J. 2005. Repbase Update, a database of eukaryotic repetitive elements. Cytogenet Genome Res 110: 462-467.

Kall L, Krogh A, Sonnhammer EL. 2004. A combined transmembrane topology and signal peptide prediction method. J Mol Biol 338: 1027-1036.

Kauffmann A, Gentleman R, Huber W. 2009. arrayQualityMetrics: a bioconductor package for quality assessment of microarray data. Bioinformatics 25: $415-416$.

Kim M, Lim JH, Ahn CS, Park K, Kim GT, Kim WT, Pai HS. 2006. Mitochondria-associated hexokinases play a role in the control of programmed cell death in Nicotiana benthamiana. Plant Cell 18: 2341-2355.

Kreuzwieser J, Scheerer U, Kruse J, Burzlaff T, Honsel A, Alfarraj S, Georgiev P, Schnitzler JP, Ghirardo A, Kreuzer I, et al. 2014. The Venus flytrap attracts insects by the release of volatile organic compounds. J Exp Bot 65: 755-766.

Krol E, Plachno BJ, Adamec L, Stolarz M, Dziubinska H, Trebacz K. 2012. Quite a few reasons for calling carnivores 'the most wonderful plants in the world'. Ann Bot 109: 47-64.

Kruse J, Gao P, Honsel A, Kreuzwieser J, Burzlaff T, Alfarraj S, Hedrich R, Rennenberg H. 2014. Strategy of nitrogen acquisition and utilization by carnivorous Dionaea muscipula. Oecologia 174: 839-851.

Lamesch P, Berardini TZ, Li D, Swarbreck D, Wilks C, Sasidharan R, Muller R, Dreher K, Alexander DL, Garcia-Hernandez M, et al. 2012. The Arabidopsis Information Resource (TAIR): improved gene annotation and new tools. Nucleic Acids Res 40: D1202-D1210.

Langmead B, Trapnell C, Pop M, Salzberg SL. 2009. Ultrafast and memoryefficient alignment of short DNA sequences to the human genome. Genome Biol 10: R25.

Leushkin EV, Sutormin RA, Nabieva ER, Penin AA, Kondrashov AS, Logacheva MD. 2013. The miniature genome of a carnivorous plant Genlisea aurea contains a low number of genes and short non-coding sequences. BMC Genomics 14: 476.

Li B, Dewey CN. 2011. RSEM: accurate transcript quantification from RNASeq data with or without a reference genome. BMC Bioinformatics 12: 323.

Libiakova M, Flokova K, Novak O, Slovakova L, Pavlovic A. 2014. Abundance of cysteine endopeptidase dionain in digestive fluid of
Venus flytrap (Dionaea muscipula Ellis) is regulated by different stimuli from prey through jasmonates. PLoS One 9: e104424.

Liu JX, Howell SH. 2010. Endoplasmic reticulum protein quality control and its relationship to environmental stress responses in plants. Plant Cell 22: 2930-2942.

Lloyd FE. 1942. The carnivorous plants. Chronica Botanica Company, Waltham, MA.

Lohse M, Nagel A, Herter T, May P, Schroda M, Zrenner R, Tohge T, Fernie AR, Stitt M, Usadel B. 2014. Mercator: a fast and simple web server for genome scale functional annotation of plant sequence data. Plant Cell Environ 37: 1250-1258.

Luo W, Friedman MS, Shedden K, Hankenson KD, Woolf PJ. 2009. GAGE: generally applicable gene set enrichment for pathway analysis. $B M C$ Bioinformatics 10: 161 .

Mithöfer A, Boland W. 2012. Plant defense against herbivores: chemical aspects. Annu Rev Plant Biol 63: 431-450.

Mittler R, Vanderauwera S, Gollery M, Van Breusegem F. 2004. Reactive oxygen gene network of plants. Trends Plant Sci 9: 490-498.

Miya A, Albert P, Shinya T, Desaki Y, Ichimura K, Shirasu K, Narusaka Y, Kawakami N, Kaku H, Shibuya N. 2007. CERK1, a LysM receptor kinase, is essential for chitin elicitor signaling in Arabidopsis. Proc Natl Acad Sci 104: 19613-19618.

Moller S, Croning MD, Apweiler R. 2001. Evaluation of methods for the prediction of membrane spanning regions. Bioinformatics 17: 646-653.

Monte I, Hamberg M, Chini A, Gimenez-Ibanez S, Garcia-Casado G, Porzel A, Pazos F, Boter M, Solano R. 2014. Rational design of a ligand-based antagonist of jasmonate perception. Nat Chem Biol 10: 671-676.

Mousavi SA, Chauvin A, Pascaud F, Kellenberger S, Farmer EE. 2013. GLUTAMATE RECEPTOR-LIKE genes mediate leaf-to-leaf wound signalling. Nature 500: $422-426$.

Myhre S, Tveit H, Mollestad T, Laegreid A. 2006. Additional gene ontology structure for improved biological reasoning. Bioinformatics 22: 2020-2027.

Nakamura Y, Reichelt M, Mayer VE, Mithofer A. 2013. Jasmonates trigger prey-induced formation of 'outer stomach' in carnivorous sundew plants. Proc Biol Sci 280: 20130228.

Noutoshi Y, Kuromori T, Wada T, Hirayama T, Kamiya A, Imura Y, Yasuda M, Nakashita H, Shirasu K, Shinozaki K. 2006. Loss of Necrotic Spotted Lesions 1 associates with cell death and defense responses in Arabidopsis thaliana. Plant Mol Biol 62: 29-42.

Paszota P, Escalante-Perez M, Thomsen LR, Risor MW, Dembski A, Sanglas L, Nielsen TA, Karring H, Thogersen IB, Hedrich R, et al. 2014. Secreted major Venus flytrap chitinase enables digestion of Arthropod prey. Biochim Biophys Acta 1844: 374-383.

Petersen TN, Brunak S, von Heijne G, Nielsen H. 2011. SignalP 4.0: discriminating signal peptides from transmembrane regions. Nat Methods 8: 785-786.

Quevillon E, Silventoinen V, Pillai S, Harte N, Mulder N, Apweiler R, Lopez R. 2005. InterProScan: protein domains identifier. Nucleic Acids Res 33: W116-W120.

Radhika V, Kost C, Boland W, Heil M. 2010. The role of jasmonates in floral nectar secretion. PLoS One 5: e9265.

Rappsilber J, Ishihama Y, Mann M. 2003. Stop and go extraction tips for matrix-assisted laser desorption/ionization, nanoelectrospray, and LC/MS sample pretreatment in proteomics. Anal Chem 75: 663-670.

Rea PA, Joel DM, Juniper BE. 1983. Secretion and redistribution of chloride in the digestive glands of Dionaea-muscipula Ellis (Venus Flytrap) upon secretion stimulation. New Phytol 94: 359-366.

Renner T, Specht CD. 2013. Inside the trap: gland morphologies, digestive enzymes, and the evolution of plant carnivory in the Caryophyllales. Curr Opin Plant Biol 16: 436-442.

Robins RJ, Juniper BE. 1980. The secretory cycle of Dionaea-muscipula Ellis. 5. The absorption of nutrients. New Phytol 86: 413-\&.

Sayers EW, Barrett T, Benson DA, Bryant SH, Canese K, Chetvernin V, Church DM, DiCuccio M, Edgar R, Federhen S, et al. 2009. Database resources of the National Center for Biotechnology Information. Nucleic Acids Res 37: D5-D15.

Scherzer S, Krol E, Kreuzer I, Kruse J, Karl F, von Ruden M, Escalante-Perez M, Muller T, Rennenberg H, Al-Rasheid KA, et al. 2013. The Dionaea muscipula ammonium channel DmAMT1 provides $\mathrm{NH}_{4}^{+}$uptake associated with Venus flytrap's prey digestion. Curr Biol 23: 1649-1657.

Scherzer S, Böhm J, Krol E, Shabala L, Kreuzer I, Larisch C, Bemm F, AlRasheid KA, Shabala S, Rennenberg H, et al. 2015. Calcium sensor kinase activates potassium uptake systems in gland cells of Venus flytraps. Proc Natl Acad Sci 112: 7309-7314.

Schulze WX, Sanggaard KW, Kreuzer I, Knudsen AD, Bemm F, Thogersen IB, Brautigam A, Thomsen LR, Schliesky S, Dyrlund TF, et al. 2012. The protein composition of the digestive fluid from the Venus flytrap sheds light on prey digestion mechanisms. Mol Cell Proteomics 11: 1306-1319.

\section{Genome Research}

www.genome.org 
Sheard LB, Tan X, Mao H, Withers J, Ben-Nissan G, Hinds TR, Kobayashi Y, Hsu FF, Sharon M, Browse J, et al. 2010. Jasmonate perception by inositol-phosphate-potentiated COI1-JAZ co-receptor. Nature 468: 400-405.

Shin H, Shin HS, Dewbre GR, Harrison MJ. 2004. Phosphate transport in Arabidopsis: Pht1;1 and Pht1;4 play a major role in phosphate acquisition from both low- and high-phosphate environments. Plant J 39: 629-642.

Simao FA, Waterhouse RM, Ioannidis P, Kriventseva EV, Zdobnov EM. 2015. BUSCO: assessing genome assembly and annotation completeness with single-copy orthologs. Bioinformatics 31: 3210-3212.

Smit AFA, Hubley R, Green P. 2013-2015. RepeatMasker Open 4.0. http:// www.repeatmasker.org.

Spurr AR. 1969. A low-viscosity epoxy resin embedding medium for electron microscopy. J Ultrastruct Res 26: 31-43.

Stephens JD, Rogers WL, Heyduk K, Cruse-Sanders JM, Determann RO, Glenn TC, Malmberg RL. 2015. Resolving phylogenetic relationships of the recently radiated carnivorous plant genus Sarracenia using target enrichment. Mol Phylogenet Evol 85: 76-87.

Thimm O, Blasing O, Gibon Y, Nagel A, Meyer S, Kruger P, Selbig J, Muller LA, Rhee SY, Stitt M. 2004. MAPMAN: a user-driven tool to display genomics data sets onto diagrams of metabolic pathways and other biological processes. Plant J 37: 914-939.

Tomatsu H, Takano J, Takahashi H, Watanabe-Takahashi A, Shibagaki N, Fujiwara T. 2007. An Arabidopsis thaliana high-affinity molybdate transporter required for efficient uptake of molybdate from soil. Proc Natl Acad Sci 104: 18807-18812.

Tran TD, Cao HX, Jovtchev G, Neumann P, Novak P, Fojtova M, Vu GT, Macas J, Fajkus J, Schubert I, et al. 2015. Centromere and telomere sequence alterations reflect the rapid genome evolution within the carnivorous plant genus Genlisea. Plant J 84: 1087-1099.

UniProt-Consortium. 2015. UniProt: a hub for protein information. Nucleic Acids Res 43: D204-D212.

Vizcaino JA, Csordas A, Del-Toro N, Dianes JA, Griss J, Lavidas I, Mayer G, Perez-Riverol Y, Reisinger F, Ternent T, et al. 2016. 2016 update of the PRIDE database and its related tools. Nucleic Acids Res 44: D447-D456.
Volkov AG, Vilfranc CL, Murphy VA, Mitchell CM, Volkova MI, O’Neal L, Markin VS. 2013. Electrotonic and action potentials in the Venus flytrap. J Plant Physiol 170: 838-846.

Wasternack C, Hause B. 2013. Jasmonates: biosynthesis, perception, signal transduction and action in plant stress response, growth and development. An update to the 2007 review in Annals of Botany. Ann Bot 111: 1021-1058.

Williams B, Verchot J, Dickman MB. 2014. When supply does not meet demand-ER stress and plant programmed cell death. Front Plant Sci 5: 211.

Wittkop T, Emig D, Lange S, Rahmann S, Albrecht M, Morris JH, Bocker S, Stoye J, Baumbach J. 2010. Partitioning biological data with transitivity clustering. Nat Methods 7: 419-420.

Xu E, Brosche M. 2014. Salicylic acid signaling inhibits apoplastic reactive oxygen species signaling. BMC Plant Biol 14: 155.

Yang DL, Yao J, Mei CS, Tong XH, Zeng LJ, Li Q, Xiao LT, Sun TP, Li J, Deng XW, et al. 2012. Plant hormone jasmonate prioritizes defense over growth by interfering with gibberellin signaling cascade. Proc Natl Acad Sci 109: E1192-E1200.

Yu G, Li F, Qin Y, Bo X, Wu Y, Wang S. 2010. GOSemSim: an R package for measuring semantic similarity among GO terms and gene products. Bioinformatics 26: 976-978.

Zaag R, Tamby JP, Guichard C, Tariq Z, Rigaill G, Delannoy E, Renou JP, Balzergue S, Mary-Huard T, Aubourg S, et al. 2015. GEM2Net: from gene expression modeling to -omics networks, a new CATdb module to investigate Arabidopsis thaliana genes involved in stress response. Nucleic Acids Res 43: D1010-D1017.

Zauber H, Schulze WX. 2012. Proteomics wants cRacker: automated standardized data analysis of LC-MS derived proteomic data. J Proteome Res 11: 5548-5555.

Received November 20, 2015; accepted in revised form April 7, 2016. 


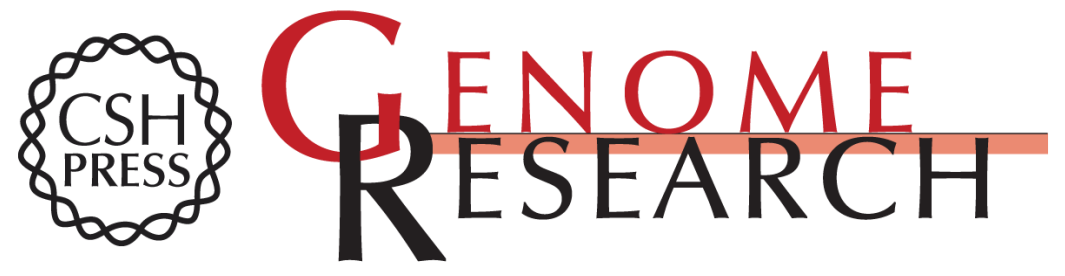

\section{Venus flytrap carnivorous lifestyle builds on herbivore defense strategies}

Felix Bemm, Dirk Becker, Christina Larisch, et al.

Genome Res. 2016 26: 812-825 originally published online May 4, 2016

Access the most recent version at doi:10.1101/gr.202200.115

\section{Supplemental} Material

References

Open Access

Creative Commons

License

Email Alerting Service
http://genome.cshlp.org/content/suppl/2016/04/28/gr.202200.115.DC1

This article cites 102 articles, 13 of which can be accessed free at: http://genome.cshlp.org/content/26/6/812.full.html\#ref-list-1

Freely available online through the Genome Research Open Access option.

This article, published in Genome Research, is available under a Creative Commons License (Attribution-NonCommercial 4.0 International), as described at http://creativecommons.org/licenses/by-nc/4.0/.

Receive free email alerts when new articles cite this article - sign up in the box at the top right corner of the article or click here.

\section{Affordable, Accurate Sequencing.}

To subscribe to Genome Research go to: https://genome.cshlp.org/subscriptions 